\title{
MEASUREMENTS OF THE EFFECTS OF STREAMWISE RIBLETS ON THE TURBULENCE STRUCTURES IN BOUNDARY LAYERS
}

by

Derek B. Ancrum

A Thesis submitted to the Faculty of Graduate and Postdoctoral Affairs

in partial fulfilment of the requirements for the degree of

\section{Master of Applied Science}

in

Aerospace Engineering

Ottawa-Carleton Institute for

Mechanical \& Aerospace Engineering

Department of Mechanical and Aerospace Engineering

Carleton University

Ottawa, Ontario

(c) 2016

Derek B. Ancrum 
The undersigned recommend to

the Faculty of Graduate and Postdoctoral Affairs

acceptance of the thesis entitled

\title{
Measurements of the effects of streamwise riblets on the turbulence structures in boundary layers
}

\author{
submitted by \\ Derek B. Ancrum \\ in partial fulfilment of the requirements for the degree of \\ Master of Applied Science in Aerospace Engineering
}

Thesis Supervisor:

Professor Metin I. Yaras

Department of Mechanical \& Aerospace Engineering

Department Chair:

Professor Ron Miller

Department of Mechanical \& Aerospace Engineering

Carleton University 


\section{Abstract}

This study presents experimental results on the effects of streamwise-oriented riblets on the coherent structures of turbulence. Hotwire measurements were performed in artificially created turbulent spots. The riblet spacings of the study correspond to 0.5 and 1.5 times the natural spacing of the low-speed streaks and fall into the category identified as wider-spaced in published literature. The cross-sectional dimensions of the riblets were chosen to promote more effective control on the development and spatial distribution of wave packets consisting of streamwise-aligned hairpin vortices.

The riblets proved to be highly effective in controlling the spatial positioning of wave packets. Of the two riblet spacings considered, the wider spacing increased the spanwise spacing of the low-speed streaks beyond their natural spacing and stabilized the wave packets over the riblet tips, enabling a reduction in their mutual interaction and realizing a reduction in their spanwise density compared to the conditions on a smooth surface. This effect may be optimized to achieve skin-friction drag and aerodynamic noise reduction. The closerspaced riblets were observed to have even more control on the spanwise positioning of the wave packets, and produced notably stronger sweep and ejection events by reducing the spanwise spacing between wave packets and promoting mutual interaction of hairpin vortices via spanwise-oriented vortical structures created by a Kelvin-Helmholtz instability mechanism. This effect may be used to achieve increased convection heat transfer in various applications without significant penalties in pressure loss. 


\section{Acknowledgments}

This work would not have been possible without the support and mentorship of my supervisor, Professor Metin I. Yaras. I would like to thank him for his patience and his ability as an educator to bring out the best in his students while always keeping their development in mind. This manuscript represents only a small portion of the knowledge I have gained working with him.

I would like to thank the machinists, administrative assistants, electricians and lab technologists who went out of their way to help whenever possible with their expertise and advice.

I would also like to thank my friends and colleagues. Truly, there is no better environment to work in than one in which helping hands (and minds) are never more than an arms length away. Thank you for your curiosity, your company and for the occasional distraction from the work at hand.

Finally, thank you to my family and my partner, Caitlin, whose endless patience and support are the reason I was able to perservere in this endeavour. 


\section{Table of Contents}

$\begin{array}{ll}\text { Abstract } & \text { iii }\end{array}$

Acknowledgments $\quad$ iv

List of Tables $\quad$ vii

$\begin{array}{ll}\text { List of Figures } & \text { viii }\end{array}$

$\begin{array}{lr}\text { List of Symbols } & 2\end{array}$

1 Background $\quad 6$

$\begin{array}{llr}2 & \text { Introduction } & 11\end{array}$

3 Experimental Setup and Data Processing 20

4 Operating Conditions and Undisturbed Boundary Layer Development 27

5 Results and Discussion 31

5.1 Turbulent spot development over a smooth surface . . . . . . . . . . . . . 31

5.2 Effects of riblets on the coherent structures of turbulence . . . . . . . . 36

6 Conclusions $\quad 45$

$\begin{array}{ll}\text { List of References } & 47\end{array}$

$\begin{array}{ll}\text { Appendix A Instrumentation Details } & 54\end{array}$ 


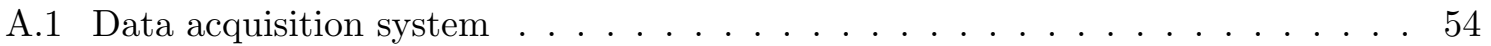

A.2 Pressure transducers . . . . . . . . . . . . . . . . . 55

A.3 Thermocouple. . . . . . . . . . . . . . . . . 57

A.4 Hotwire anemometer . . . . . . . . . . . . . . . . . 58

\section{$\begin{array}{lll}\text { Appendix B Wind Tunnel Commissioning } & 61\end{array}$}

B.1 Traverse system commissioning . . . . . . . . . . . . . . . . 62

B.2 Contraction outlet flow commissioning with an empty test section . . . . . . . 63

B.2.1 Contraction wall boundary layer at the outlet . . . . . . . . . . 64

B.2.2 Contraction outlet flow uniformity . . . . . . . . . . . . 65

B.2.3 Flow alignment . . . . . . . . . . . . . . . . 65

B.2.4 Freestream turbulence . . . . . . . . . . . . . . . . 66

B.3 Benchmark testing . . . . . . . . . . . . . . . . . . . . . 69

$\begin{array}{lll}\text { Appendix C Additional Information on the Experimental Setup } & 71\end{array}$

C.1 Surface manufacturing . . . . . . . . . . . . . . . . . . 71

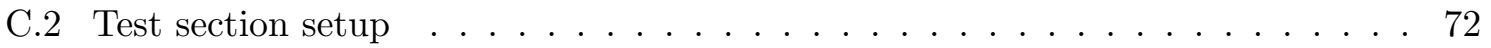

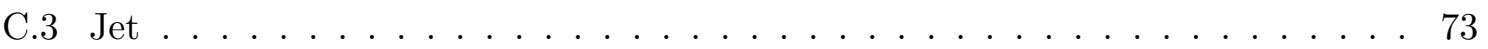




\section{List of Tables}

A.1 A/D Conversion Resolution . . . . . . . . . . . . . . . 54

A.2 Pressure transducer calibration coefficients . . . . . . . . . . 56

B.1 Traverse system commissioning data . . . . . . . . . . . . . 62 


\section{List of Figures}

1.1 Smoke-wire visualization of lambda-vortices, hairpin vortices and turbulent spots; adapted from Perry et al. $(1981) \ldots \ldots \ldots \ldots$. . . . . . . . 7

2.1 Schematic of a hairpin vortex . . . . . . . . . . . . . . . . . 12

2.2 Schematic of secondary flows within streamwise grooves of a closely-spaced riblet surface driven by the interaction of the hairpin vortex legs with the riblets 17

2.3 Formation of vortices spanning multiple riblet grooves through K-H instability 18

3.1 Test section configuration . . . . . . . . . . . . . . . . . 21

3.2 Riblet geometry . . . . . . . . . . . . . . . . . . . . 22

4.1 Undisturbed boundary-layer velocity profiles for $R e_{L}=3.1 \times 10^{5}$ (a,c,e) and $R e_{L}=2.2 \times 10^{5}(\mathrm{~b}, \mathrm{~d}, \mathrm{f})$. For the riblet surfaces, the dashed horizontal line identifies the location of the riblet tip. . . . . . . . . . . . . . 29

5.1 Perturbation velocity in the $\mathrm{z}$-t plane on the smooth surface measured at $\mathrm{x}=600$ mm for a) $R e_{L}=2.2 \times 10^{5}$ and b) $R e_{L}=3.1 \times 10^{5} \ldots \ldots 33$

5.2 Perturbation velocities in the $\mathrm{y}-\mathrm{z}$ plane on the smooth surface measured at $\mathrm{x}=600 \mathrm{~mm}$ for a) $R e_{L}=2.2 \times 10^{5}$ and b) $R e_{L}=3.1 \times 10^{5} \ldots \ldots 34$

5.3 Perturbation velocity distribution in the $\mathrm{y}$-t plane over the smooth surface measured at $\mathrm{x}=600 \mathrm{~mm}$ at a) $\left.\left.z / \delta_{\text {ref }}^{*}=0.0, \mathrm{~b}\right) z / \delta_{\text {ref }}^{*}=10.0, \mathrm{c}\right) z / \delta_{\text {ref }}^{*}=20.0$,

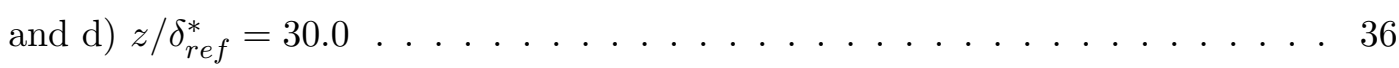

5.4 Variation of the spot propagation parameter for $R e_{L}=3.1 \times 10^{5}$ over the

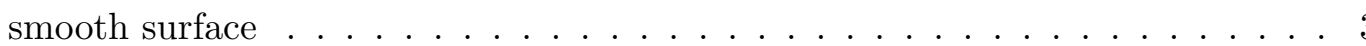


5.5 Comparison of the ensemble-averaged streamwise velocity and spanwise vorticity at $\mathrm{x}=600 \mathrm{~mm}$ and $R e_{L}=3.1 \times 10^{5}$ between the smooth surface and the a) closer-spaced riblet; groove center b) closer-spaced riblet; riblet tip c) wider-spaced riblet; groove center d) wider-spaced riblet; riblet tip . . . . . . . 38

5.6 Perturbation velocity distribution in the $y-z$ plane a) smooth surface b) surface with closer-spaced riblets c) surface with wider-spaced riblets . . . . . . . . 39

5.7 Schematic of a hairpin vortex and its induced motions over an isolated riblet. The red and blue regions denote regions of positive and negative perturbation velocities, respectively . . . . . . . . . . . . . . . 40

5.8 Topology of the turbulent spot developing over the wider-spaced riblet surface at a) $y / \delta_{\text {ref }}^{*}=8.8$ and b) $y / \delta_{\text {ref }}^{*}=2.3\left(R e_{L}=3.1 \times 10^{5}, \mathrm{x}=600 \mathrm{~mm}\right) \ldots . .41$

5.9 Topology of the turbulent spot over the closer-spaced riblet surface at a) $y / \delta_{\text {ref }}^{*}=8.8$ and b) $y / \delta_{\text {ref }}^{*}=2.3\left(R e_{L}=3.1 \times 10^{5}, \mathrm{x}=600 \mathrm{~mm}\right) \ldots \ldots . . .42$

5.10 Perturbation velocity of the turbulent spot in the $y$-t plane on the closer-spaced riblet surface $\left(R e_{L}=3.1 \times 10^{5}, \mathrm{x}=600 \mathrm{~mm}\right) \ldots \ldots \ldots \ldots$. . . . . . 42

5.11 Schematic of two spanwise adjacent wave packets interacting with each other and the prevailing spanwise vortices over the surface with closer-spaced riblets

5.12 Streamwise variation of the spot propagation parameter over all three test surfaces at both flow Reynolds numbers . . . . . . . . . . . . . . 44

A.1 Thermocouple calibration . . . . . . . . . . . . . 57

A.2 Results of 16 calibrations of the hotwire sensor approximated by a 9th-order polynomial fit, each colour represents one calibration curve . . . . . . . 59

A.3 Probe angle sensitivity study for a) $R e_{L}=2.2 \times 10^{5}$ and b) $R e_{L}=3.1 \times 10^{5}$ at $\mathrm{x}=600 \mathrm{~mm}, \mathrm{z}=0 \mathrm{~mm}$ on the closer-spaced riblet surface . . . . . . . . 60

B.1 Schematic of Carleton University's low-turbulence, open-circuit, suction wind

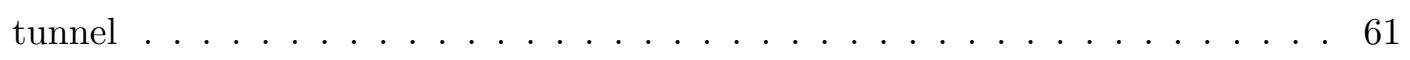

B.2 Positioning of the wind tunnel within the Laboratory . . . . . . . . . . . 63

B.3 Shadowgraph of the a) Standard Mosquito Mesh and b) Shade Screen . . . . . 64 
B.4 Flow uniformity at the contraction outlet for a) $4.5 \mathrm{~m} / \mathrm{s}$ and b) $9.0 \mathrm{~m} / \mathrm{s}$ cen-

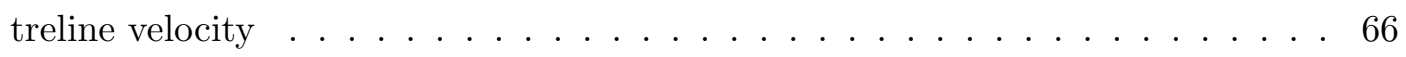

B.5 Contraction outlet flow alignment in a) pitch and b) yaw for $9.0 \mathrm{~m} / \mathrm{s} \ldots \ldots 7$

B.6 Contraction outlet flow alignment in a) pitch and b) yaw for $4.5 \mathrm{~m} / \mathrm{s} \ldots$. . . . 68

B.7 Contraction outlet freestream turbulence intensity at various wind tunnel cen-

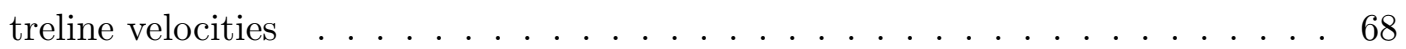

B.8 Benchmark measurement of the Blasius boundary layer at a) $2.2 \mathrm{~m} / \mathrm{s}$, b) 4.6

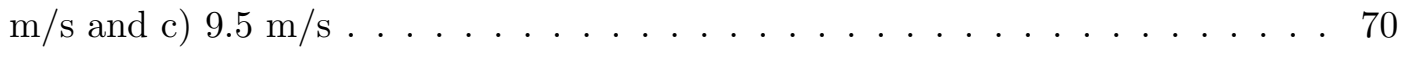

C.1 Anemometer signal of jet measurement $25 \mathrm{~mm}$ above the baseline surface . . . 74 


\section{Preface}

The content of this thesis is the reproduction of the journal paper listed below, which is currently under review by the journal. This paper was reformatted to meet the thesis requirements at Carleton University and a background chapter was added to provide the nonexpert reader with adequate background on the topics discussed herein. Readers who wish to cite sections of this thesis are strongly encouraged to cite this journal paper following its publication.

Ancrum, D.B. and Yaras, M.I. (2016). Measurements of the effects of streamwise riblets on the turbulence structures in boundary layers. Submitted to ASME J. of Fluids Engineering. 


\title{
Nomenclature
}

\author{
$A / D \quad$ analog-to-digital \\ $A_{g}^{+} \quad$ cross-sectional area of the riblet groove in wall-units \\ $D A Q \quad$ data acquisition system \\ $d \quad$ dimension on riblet surface $(\mathrm{mm})$ (Fig. 3.2) \\ DNS direct numerical simulation \\ $E_{w} \quad$ temperature corrected hotwire signal (V) \\ $E_{w, r} \quad$ raw hotwire signal (V) \\ $k \quad$ thermal conductivity $\left(\frac{W}{m K}\right)$ \\ $K-H \quad$ Kelvin-Helmholtz \\ $l_{g}^{+} \quad$ riblet sizing parameter, $l_{g}^{+}=\left(A_{g}^{+}\right)^{1 / 2}$ \\ $L \quad$ length of the test surface \\ $L_{x} \quad$ streamwise size of the turbulent spot $(\mathrm{mm})$ \\ $L_{z} \quad$ spanwise size of the turbulent spot $(\mathrm{mm})$
}


$R e_{x} \quad$ Reynolds number based on the streamwise distance from the leading edge and the freestream velocity $25 \mathrm{~mm}$ downstream of leading edge, $25 \mathrm{~mm}$ above the surface and at the spanwise position of the jet

$R e_{L} \quad$ Reynolds number based on the length of the test surface and the freestream velocity $25 \mathrm{~mm}$ downstream of leading edge, 25 $\mathrm{mm}$ above the surface and at the spanwise position of the jet

$R e_{\delta^{*}} \quad$ Reynolds number based on the local boundary-layer displacement thickness and the local freestream velocity, $u_{e}$

$t \quad$ time $(\mathrm{s})$

$T_{a} \quad$ ambient temperature $(\mathrm{K})$

$T_{a, r} \quad$ reference ambient temperature $(\mathrm{K})$

$T_{w} \quad$ wire temperature $(\mathrm{K})$

$T-S \quad$ Tolmien-Schlichting

$u \quad$ instantaneous streamwise velocity $(\mathrm{m} / \mathrm{s})$

$\bar{u} \quad$ time-averaged streamwise velocity $(\mathrm{m} / \mathrm{s})$

$\tilde{u} \quad$ ensemble-averaged streamwise perturbation velocity nondimensionalized by $u_{e}$ (Eq. 3.1)

$\hat{u} \quad$ ensemble-averaged streamwise velocity $(\mathrm{m} / \mathrm{s})$

$u^{\prime} \quad$ root-mean-square streamwise velocity fluctuations nondimensionalized by $u_{e}$ (Eq. 3.2)

$u_{e} \quad$ local boundary-layer edge velocity $(\mathrm{m} / \mathrm{s})$ 


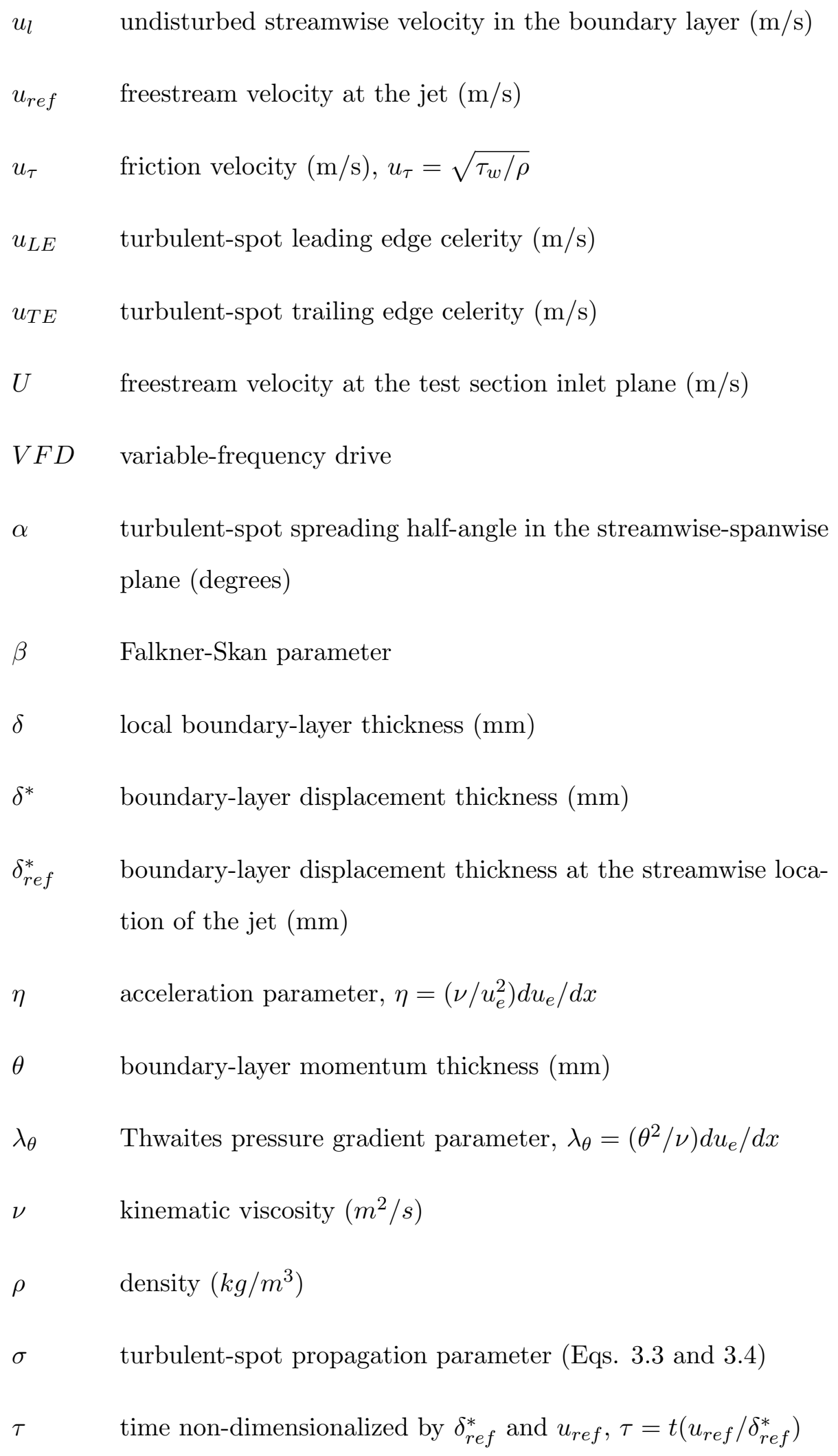


$\tau_{w} \quad$ wall shear stress $\left(N / m^{2}\right)$

$\phi \quad$ riblet side-wall angle (degrees)

$\omega_{z} \quad$ ensemble-averaged spanwise component of vorticity $(1 / \mathrm{s})$ 


\section{Chapter 1}

\section{Background}

This chapter is intended for readers unfamiliar with transitioning and turbulent boundary layers and their underlying coherent structures. Readers familiar with these subjects may begin their reading with Chapter 2 which introduces subjects more specific to this study.

Laminar and turbulent boundary layers have different properties which can make either promoting or delaying the transition from laminar to turbulent state desirable depending on the application. A turbulent boundary layer can increase surface heat transfer and is more resistant to separation but also increases skin-friction drag and flow-induced noise when compared to a laminar boundary layer.

The transition of boundary layers has been observed to occur through either natural or bypass modes. Natural transition, visualized in Fig. 1.1, occurs in low-disturbance environments and is characterized by the appearance of viscous instabilities. When the boundary layer reaches a critical Reynolds number, these instabilities cause streamwise grouping of the boundary-layers' spanwise vorticity which grow to form Tolmien-Schlichting (T-S) waves. In the linear-growth stage of these waves, their amplitude grows linearly on a logarithmic scale as they propagate downstream (Reed \& Saric, 1996). Once the wave amplitude has increased to about $1 \%$ to $2 \%$ of the local freestream velocity, the T-S waves enter a phase of nonlinear growth (Reed \& Saric, 1996). This phase is initiated by a secondary instability that causes an oscillation in the streamwise-spanwise plane. The streamwise component of the grouped vorticity induces a spanwise-non-uniform wall-normal motion which lifts the crests of the waves away from the surface to form lambda-shaped vortices (Asai \& Nishioka, 1989; 


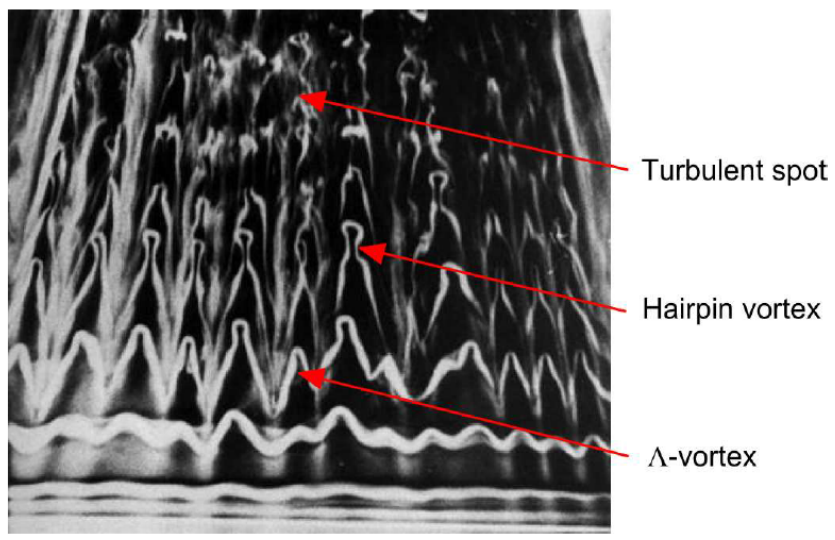

Figure 1.1: Smoke-wire visualization of lambda-vortices, hairpin vortices and turbulent spots; adapted from Perry et al. (1981)

Kachanov, 1994). The streamwise-oriented segments of the lambda-shaped vortices continue to induce wall-normal motions which lift the heads of the vortices away from the wall where they convect downstream faster, stretching the vortices into a hairpin shape. These vortical structures are the fundamental flow structures in turbulent boundary layers and have been observed to form in a range of shapes including symmetric, asymmetric, hairpin, horseshoe and lambda-shaped vortices. For brevity, all configurations will be identified herein as hairpin vortices. Hairpin vortices grow and generate new vortices in groups known as wave packets through a process described in Chapter 2. The formation of these wave packets represents the initial breakdown of a shear layer into a self-regenerative turbulent state. Once several generations of wave packets are created, they form a localized region of turbulent flow conventionally referred to as a turbulent spot. In natural transition, several turbulent spots form at a preferred streamwise location at predictable rates of formation in the spanwise direction and time, and through lateral and longitudinal growth as they convect downstream eventually merge with each other to create a fully turbulent boundary layer.

In flows with large disturbances, the boundary layer undergoes bypass transition which skips the linear-growth and secondary instability phases of natural transition. Instead, the 
transition process is characterized by the linear, transient growth of streaks within the streamwise velocity field of the laminar boundary layer. When these streaks reach a critical amplitude, they develop secondary instabilities which lead to the breakdown into turbulence. In naturally occurring flows, this mode of transition can be triggered by environmental disturbances such as freestream turbulence, surface roughness, surface vibration or sound waves. In experimental studies, many different techniques are used to achieve controlled bypass transition, including electric discharge (Wygnanski et al., 1976), roughness elements (Zhong et al., 1998) and wall-normal jets (Yaras, 2007). Depending on the strength of the disturbance, one or multiple hairpin vortices will form surrounding the streaks through the inviscid instability of the shear layer, and will eventually develop into a fully formed turbulent spot (Singer \& Joslin, 1994; Katz et al., 1990). The characteristics of a turbulent spot are independent of the source of the initial disturbance once it is fully developed (Schubauer \& Klebanoff, 1956).

Turbulent spots are traditionally considered in the context of boundary-layer transition; however, they are also relevant to the physics of fully-turbulent boundary layers. Turbulent spots exhibit many of the transient flow characteristics of a fully turbulent boundary layer albeit with fewer and more spatially organized coherent structures which exist within a narrower range of scales (Sabatino \& Smith, 2008). This allows for a clearer examination of the cause-and-effect relationships during the generation and growth of individual flow structures in turbulent spots. The growth, propagation and topology of turbulent spots have been well documented through experiments (e.g. Schubauer \& Klebanoff, 1956; Wygnanski et al., 1976; Gadelhak et al., 1981; Amini \& Lespinard, 1982; Gutmark \& Blackwelder, 1987; Schröder \& Kompenhans, 2004; Yaras, 2007) and simulations (e.g. Singer \& Joslin, 1994; Johnson, 2001; Brinkerhoff \& Yaras, 2014).

When viewed in the streamwise-spanwise plane, a typical turbulent spot has a curved arrowhead shape. The tip of the arrowhead has been observed to point in either the upstream or downstream direction depending on whether the disturbance creating the spot originated from the upper or lower part of the boundary layer respectively, with the former being dominant in cases where the disturbance spans the boundary layer (Wu et al., 1999; Yaras, 2007). Independent of its orientation, a turbulent spot tends to be symmetric about its centreline 
and has a typical spreading half angle of between 6 and 10 degrees as affected by the relative rates of longitudinal and spanwise growth of the spot as it propagates downstream (Singer \& Joslin, 1994; Wygnanski et al., 1976). The longitudinal growth is driven in part by the mean shear of the boundary layer which causes the leading and trailing edges to propagate at different rates relative to the freestream flow. The leading edge, which consists of the heads of large hairpin vortices and reaches well into the freestream, typically has a celerity of $0.95 U_{0}$ while the trailing edge, which consists of coherent structures closer to the wall, has a typical celerity of $0.5 U_{0}$ (Brinkerhoff \& Yaras, 2014). The spreading rates and celerities of the turbulent spot are affected by several flow parameters including freestream turbulence intensity and length scale, streamwise pressure gradient, streamline curvature and surface roughness (Roberts \& Yaras, 2005).

The leading edge of the turbulent spot consists of the spanwise oriented vorticity of the heads of large, stretched hairpin vortices which lie above a region of laminar flow (Gutmark \& Blackwelder, 1987). These large structures grow as the spot moves downstream due to the viscous diffusion of their vorticity and the entrainment of low speed fluid from the laminar region beneath the structures (Brinkerhoff \& Yaras, 2014). The entrained laminar fluid is eventually swept into the main body of the turbulent spot (Singer \& Joslin, 1994; Johnson, 2001). The main body is densely populated with several generations of hairpin vortices of various scales and configurations. Mature hairpin vortices extend into the freestream and travel faster relative to younger vortices which exist closer to the surface. This difference in celerity can lead to cut, connect and pairing processes which can occur as the larger hairpin vortices overtake the smaller ones. The final distinct region associated with a turbulent spot consists of a region of stable, laminar flow containing persistent high-speed streaks which forms in the wake of the turbulent spot (Brinkerhoff \& Yaras, 2014).

Many techniques have been developed to control turbulent and transitioning boundary layers both through active and passive means. Active control requires that external energy be expended and includes techniques such as plasma actuators (Samimy et al., 2007) and oscillating walls (Choi \& Clayton, 1998). Passive control techniques require no application of external energy. Examples of passive control techniques include surface riblets (Choi et al., 
1993; Garcia-Mayoral \& Jimenez, 2011a; Goldstein et al., 1995; Bechert et al., 1997), compliant coatings (Choi et al., 1997; Dixon et al., 1994), slip walls (Bechert et al., 1996) and permeable walls (Suga et al., 2011). These techniques typically aim to reduce skin friction or delay the onset of transition, and have achieved more modest results to date compared to active control techniques. The present work investigates the underlying physics of boundary layer turbulence when affected by streamwise riblets. The results of this investigation are intended to provide guidance for further design optimization of riblets to improve their effectiveness in controlling boundary layer turbulence. 


\section{Chapter 2}

\section{Introduction}

Controlling boundary-layer transition and turbulence can lead to improvements in lift, drag and flow-induced noise characteristics (Bushnell \& McGinley, 1989). This potential for improved performance has generated significant interest in the development of passive and active flow control techniques which aim to reduce turbulence by affecting the onset and/or rate of transition and the turbulence characteristics of the fully-turbulent boundary layer (Bushnell, 2003).

Turbulent and transitioning boundary layers are densely populated with self-regenerating coherent flow structures (Perry et al., 1981; Schröder \& Kompenhans, 2004). These structures have been observed to form in several configurations including symmetric, asymmetric, hairpin, horseshoe and lambda-shaped vortices. For brevity, all configurations will be identified herein as hairpin vortices, the topology of which is depicted in Fig. 2.1. The local flow environment induced by a hairpin vortex promotes the wall-normal redistribution of fluid within the boundary layer through upwash and downwash motions induced locally by its quasi-streamwise-oriented legs. These motions cause low-speed fluid to move away from the wall between the legs of the hairpin vortex and high-speed fluid to move toward the wall on the outboard side of the legs, leading to the formation of streamwise-oriented streaks of lowand high-speed flow relative to the surrounding fluid (Fig. 2.1) (Schröder \& Kompenhans, 2004; Yaras, 2007). The shear layer surrounding the low-speed streak is inviscid-unstable and causes streamwise grouping of vorticity to occur at a dominant wavelength when the velocity deficit reaches a threshold magnitude (Singer \& Joslin, 1994; Brinkerhoff \& Yaras, 2014). This 


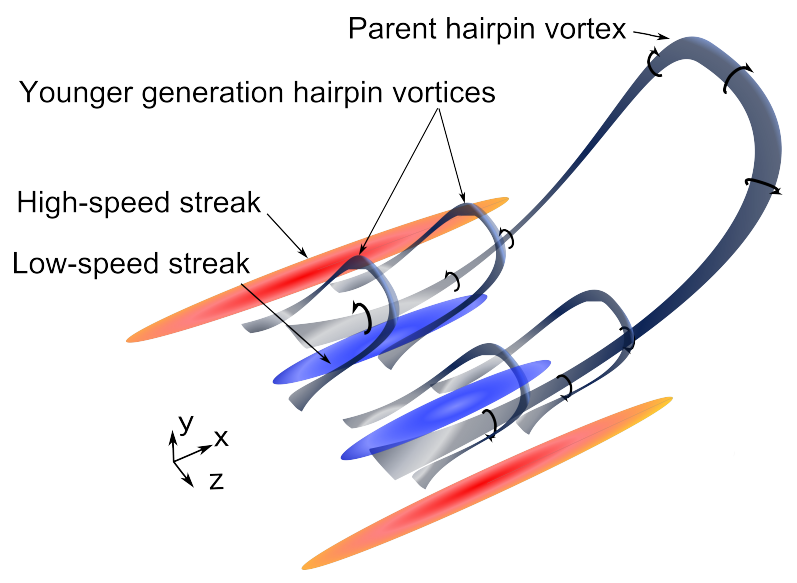

Figure 2.1: Schematic of a hairpin vortex

causes the low-speed streaks to undergo a secondary instability and deform in either a sinuous or varicose pattern as discussed in more detail later in this section.

The vortices which result from the streamwise grouping of vorticity form groups of wallnormal-oriented vorticity loops which surround the low-speed streak and straddle a single leg of the hairpin vortex. These loops eventually stretch and grow to form streamwise aligned groups of hairpin vortices called wave packets (Zhou et al., 1999). These hairpin vortices may merge with those on the adjacent leg of the parent hairpin vortex if the parent has streamwise oriented legs that are in relatively close proximity to one another. Wave-packets can thus form straddling either one or both legs of the parent hairpin vortex (Brinkerhoff \& Yaras, 2014). The number of hairpin vortices within a wave packet has been observed to vary with Reynolds number from two or three hairpin vortices at $R e_{\delta^{*}} \sim O\left(10^{2}\right)$ to four or five hairpin vortices at $R e_{\delta^{*}} \sim O\left(10^{3}\right)$, where $R e_{\delta^{*}}$ is the Reynolds number based on the local freestream velocity and displacement thickness of the boundary layer (Adrian et al., 2000). The streamwise spacing of hairpin vortices within a wave packet in a zero-pressure-gradient boundary layer is related to the dominant wavelength of instability of the local shear layer surrounding the low-speed streak and is characteristically on the order of several hundred wall-units (Adrian et al., 2000). The wall-unit refers to the length scale that is defined by the kinematic viscosity of the fluid and the friction velocity at the nearest wall. The friction velocity, $u_{\tau}=\sqrt{\tau_{w} / \rho}$, is calculated using the shear stress and density at the wall. In the 
spanwise direction, adjacent wave packets have a mean spacing which lies along a lognormal probability distribution with a dense range of between 60 to 180 wall-units and a mean value of 100 wall units, independent of flow Reynolds number (Smith \& Metzler, 1983; Kline et al., 1983; Adrian et al., 2000). The natural spanwise spacing of the hairpin vortices corresponds to the wavelength of perturbations that yields the highest growth rate in the kinetic energy of the perturbations over a period not greater than the local eddy-turnover time, and represents a balance of viscous dissipation and the kinetic energy of the transient wall-normal motions (Butler \& Farrell, 1993; Baig \& Chernyshenko, 2004). The spanwise spacing of the hairpin vortices has been observed to increase under two conditions. The first condition occurs as the heads of the older generations of hairpin vortices extend higher in the boundary layer where the population of hairpin vortices is less dense (Smith \& Metzler, 1983). The second condition occurs if the boundary layer is subjected to a favourable pressure gradient. In the latter case, the spanwise spacing between wave packets has been found to be two to three times larger than the average for a zero-pressure-gradient boundary layer (Yaras, 2007).

As a wave packet develops, the hairpin vortices within it grow through both self-induced motions and interactions with neighbouring hairpin vortices. The streamwise component of vorticity in the legs of the hairpin vortex induce an upward motion which lifts the spanwiseoriented head away from the surface where it is carried downstream faster due to the mean shear in the boundary layer. This difference in velocity between the head and legs of the hairpin vortex causes it to stretch in the streamwise direction thereby increasing its vorticity through conservation of angular momentum. Hairpin vortices can also grow by interacting with structures in neighbouring wave packets. This interaction can lead to the breakdown of the legs of the interacting hairpin vortices into smaller vortical structures due to their opposite directions of rotation while the heads merge to form a hairpin vortex of greater spanwise extent (Adrian et al., 2000; Tomkins \& Adrian, 2003). The amalgamation and self-induced growth of hairpin vortices creates a flow topology where wave packets of different scales reside within each other, with the smallest wave packets situated near the wall and the largest wave packets extending away from the wall up to a distance of several times the boundary-layer thickness, where the boundary layer thickness is defined as the wall-normal location where the streamwise 
velocity component reaches $99 \%$ of the freestream value (Adrian et al., 2000). The hairpin vortices within the larger wave packets convect faster than their near-surface counterparts. This can cause the larger, faster hairpin vortices to overtake smaller ones, leading to vortical dynamics that may involve cut, connect, and pairing processes when multiple generations of wave packets co-exist (Zhou et al., 1999; Adrian et al., 2000; Panton, 2001; Smits \& Delo, 2001).

The formation of hairpin vortices in the shear layer surrounding the low-speed streaks is coupled to the streak deformation through secondary instabilities. These instabilities occur in two modes: a symmetric varicose mode which is observed as streamwise-periodic widening and narrowing of the streak and an asymmetric sinuous mode which is observed as an oscillation of the streaks in the streamwise-spanwise plane. Experiments by Asai et al. (2002) in which low-speed streaks were artificially disturbed to produce each instability mode found that the instability mode that is most likely to occur depends on the width of the streak, magnitude of the velocity deficit in the streak, and the excitation of the streak by external disturbances. Varicose mode was found to be dominant for wider streaks with a larger velocity deficit whereas weaker and narrower streaks were more susceptible to sinuous instability. Hack \& Zaki (2014) performed a statistical analysis of streak instabilities based on direct numerical simulations of zero-pressure-gradient and adverse-pressure-gradient boundary layers. Through this study it was determined that sinuous instability is caused by an outer mode in which the low speed streak is lifted towards the edge of the boundary layer and is deformed by freestream disturbances. Sinuous instability was found to occur in $90 \%$ of streaks in a zeropressure-gradient boundary layer. Varicose instability was found to occur more frequently in adverse-pressure-gradient boundary layers and is created through the inner-mode instability. The inner-mode instability occurs when a high-speed streak overlaps with the trailing edge of a low-speed streak causing the wall-normal velocity profile to become inflectional and thus inviscid-unstable, leading to the secondary instability of the low-speed streak. In lowdisturbance, favourable-pressure-gradient boundary layers with streamwise acceleration rates consistent with a re-laminarizing flow, the low-speed streaks have been found to be less prone to secondary instabilities (Piomelli et al., 2000). The stabilization effect on the low-speed 
streaks is caused by the damping of the wall-normal and spanwise fluctuation velocities, and is thought to be an important mechanism for the re-laminarization of a turbulent boundary layer under these conditions (Piomelli \& Yuan, 2013). Conversely, secondary streak instabilities have been found to occur much more frequently in flows with high levels of external disturbances (Yaras, 2007).

Several technologies have been developed to control skin-friction, flow-induced noise and surface convection heat transfer through the control of the turbulence regeneration process in the boundary layer. One of the most successful passive techniques for controlling turbulence is the installation of streamwise oriented riblets on the surface. Such surfaces consist of streamwise-aligned grooves with rectangular, triangular or hemispherical cross-sections. While riblets with a long spanwise wavelength have been shown analytically to produce a drag reduction in laminar flows (Mohammadi \& Floryan, 2013a,b), the majority of published work on this subject has instead focused on the riblets' ability to reduce skin-friction drag in transitioning and turbulent boundary layers. In this context, several geometric parameters including the width of the riblet tip, the riblet height, and the width of the groove have been identified as having an impact on the ability of the riblets to influence the turbulence regeneration process. The width of the riblet tip is found to be most effective in controlling turbulence when it is made as narrow as possible (Bechert et al., 1997). The most important dimensions dictating the aerodynamic effect of riblets on boundary-layer turbulence are the riblet height and riblet spacing which, unless otherwise specified, is defined as the center-tocenter distance between riblets. Riblets have been studied in both closely-spaced and widelyspaced configurations resulting in net drag reduction and drag increase, respectively (Bechert et al., 1997). Closely-spaced riblets typically have spacings in the range of 15-25 wall-units, corresponding to about one-third to one-half of the diameter of a typical quasi-streamwisealigned leg of a mature hairpin vortex. Such riblets have been found to be most effective when their height is about half of their spanwise spacing (Bechert et al., 1997). Widely-spaced riblets are defined as those with spacing larger than the aforementioned optimum range for drag reduction (Goldstein et al., 1995). The effectiveness of the riblets at controlling turbulence has been suggested to be insensitive to the cross-sectional shape of the riblets and hence of the 
grooves (Bechert et al., 1997). However, the cross-sectional area of the groove is an important parameter in predicting whether the riblet will enhance or diminish the effects of turbulence. Garcia-Mayoral \& Jimenez (2011b) characterized the optimum geometry for drag reduction using the parameter $l_{g}^{+}=\left(A_{g}^{+}\right)^{1 / 2}$, where $A_{g}^{+}$is the cross-sectional area of the groove in wall-units. These authors suggest that optimum drag reduction occurs at $l_{g}^{+}=10.7 \pm 1$, with values larger than the optimum corresponding to the drag-increasing, widely-spaced riblets.

Both the closely-spaced and widely-spaced riblet configurations add a significant amount of wetted area to an otherwise smooth surface. Closely-spaced riblets produce a net drag reduction despite the increased wetted surface area because their smaller groove width hinders the development of spanwise flow within the groove, thereby forcing the legs of the hairpin vortices to reside above the wall-normal position of the riblet tips (Choi et al., 1993; Lee \& Lee, 2001). The elevated wall-normal position of the hairpin vortices creates a corresponding wall-normal shift in the position of high-speed streaks and constrains the high-speed-streak driven increase in wall shear primarily to the small surface area at the tips of the riblets (Choi et al., 1993). The spanwise-moving fluid beneath the streamwise legs of the elevated hairpin vortex interacts with the riblet tips to form secondary flows as shown in Fig. 2.2 (Klumpp et al., 2010). These smaller streamwise vortices interact with and weaken the legs of the hairpin vortices (Klumpp et al., 2010). This results in high-speed streaks with reduced velocity excess, thus contributing to the reduction in the wall shear stress. The weakened hairpin vortices also produce weaker upwash events, thereby reducing the magnitude of the velocity deficit in the low-speed streaks (Chernorai et al., 2006; Litvinenko et al., 2006; Boiko, 2007). The shear layer surrounding the weakened streaks is less prone to developing secondary instabilities which inhibits the regeneration of hairpin vortices (Boiko, 2007; Chernorai et al., 2006; Ma et al., 2005). This turbulence-inhibiting effect of closely spaced riblets is also evident through reduced spreading rates of turbulent spots. For example, Strand \& Goldstein (2011) measured a $14 \%$ reduction in the spot spreading rate due to the presence of closely-spaced surface riblets.

Widely-spaced riblets do not exert the same level of control on the spanwise flow induced by the streamwise oriented legs of the hairpin vortices (Goldstein \& Tuan, 1998). The spacing 


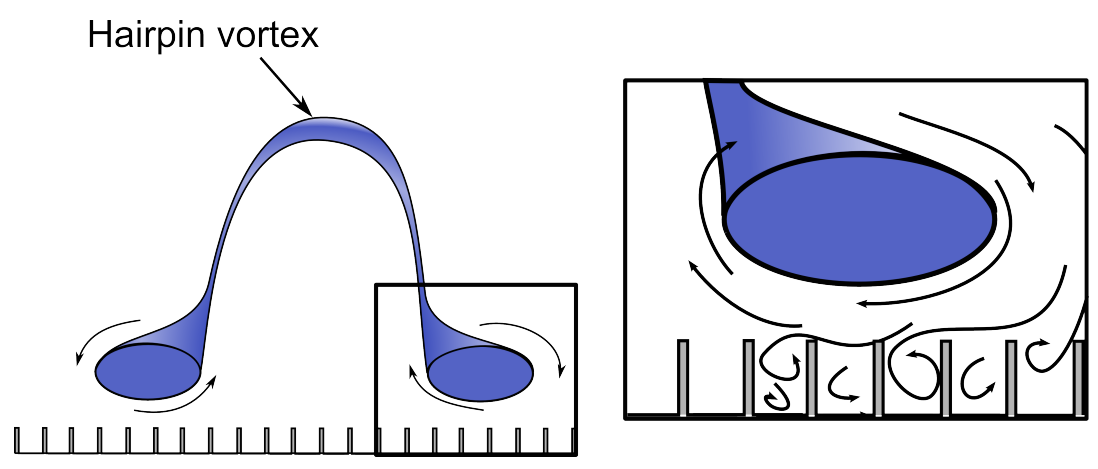

Figure 2.2: Schematic of secondary flows within streamwise grooves of a closely-spaced riblet surface driven by the interaction of the hairpin vortex legs with the riblets

between riblets of this type allows the spanwise flow to develop to a greater extent than over closely-spaced riblets such that the streamwise legs of the hairpin vortices are able to settle within the grooves, negating the turbulence reducing effects observed over the closely-spaced riblets. In a DNS study by Goldstein \& Tuan (1998), a tendency for the hairpin vortices to reside straddling the riblet tips was observed over widely-spaced riblets with a spacing of 65.3 wall-units. The authors noted that this tendency was transient and the mean streak spacing did not increase to match the riblet spacing. Goldstein \& Tuan (1998) also observed this effect for riblets spaced 375 wall-units apart, which were effectively aerodynamically isolated from one another. This suggests that not only the spacing of the riblets but the effect of their geometry on the surrounding flow-field dictates the preferred spanwise positioning of the hairpin vortices.

The location of hairpin-vortex legs within the groove causes the high-speed streaks to form in close proximity of the base and side-walls of the groove and increases the strength of ejection events as the induced spanwise flow encounters the riblets and is forced upwards (Goldstein \& Tuan, 1998). The increase in skin-friction drag of widely-spaced riblets compared to closelyspaced riblets and smooth surfaces has typically been attributed to the enhanced strength of these ejections and the increase in surface area (Choi et al., 1993; Lee \& Lee, 2001). DNS studies by Garcia-Mayoral \& Jimenez (2011a) have since found that the increase in skinfriction drag may be due in part to the formation of quasi-two-dimensional spanwise vortices which span over several riblets and remain coherent despite being disrupted by the riblet tips. 
These flow structures are similar to those which have been observed to be the dominant cause of increased turbulence over porous surfaces (Suga et al., 2011). They are formed through the inviscid Kelvin-Helmholtz (K-H) instability mode which occurs due to an inflection point in the streamwise velocity distribution casued by the relaxation of the impermeability condition of the plane at the wall-normal position of the riblet tips as depicted in Fig. 2.3 (GarciaMayoral \& Jimenez, 2011a; Suga et al., 2011). The spanwise vortices increase the wall-normal mixing due to locally induced ejection and sweep motions. They are observed to begin to form on surfaces with riblets that are optimized in height and spacing for drag-reduction and rapidly increase in size and strength with increased riblet height. The sense of rotation of the spanwise vortices described by Garcia-Mayoral \& Jimenez (2011a) is the same as that of the hairpin vortices observed to form over the riblet tips by Goldstein \& Tuan (1998). The interaction between these structures has not been documented. However, their independent observation over similarly sized riblets indicate that they may interact constructively or at least neutrally in producing the net increase in skin-friction drag observed over widely-spaced riblets.

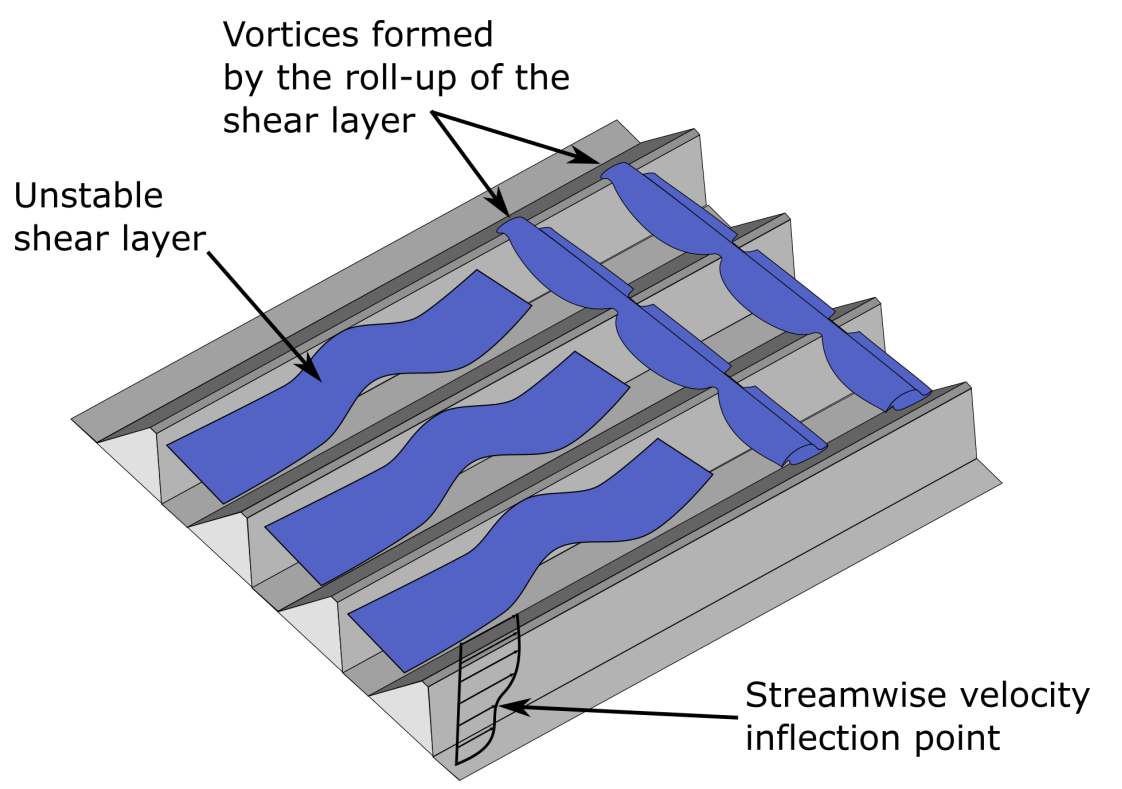

Figure 2.3: Formation of vortices spanning multiple riblet grooves through K-H instability 
The effects of both closely- and widely-spaced riblets on flow-induced noise and forcedconvection heat transfer is not as well documented as their effects on skin-friction drag. Modest improvement in noise levels have been observed over closely-spaced riblets which is likely due to the reduction in the perturbation velocities and associated pressure fluctuations induced by the weaker hairpin vortices (Lancey \& Reidy, 1989; Keith, 1989). Weaker hairpin vortices also result in reduced wall-normal mixing and hence reduced convection heat transfer on surfaces with closely-spaced riblets (Stalio \& Nobile, 2003). Improvements in convection heat transfer have been observed for widely-spaced riblets with heights on the order of the height of the thermal layer thickness (Stalio \& Nobile, 2003). The mechanism of improved heat transfer for widely-spaced riblets is primarily attributed to the increase in surface area by Stalio \& Nobile (2003), however given the observations by Garcia-Mayoral \& Jimenez (2011a), the increase in mixing produced by the spanwise vortices which form over the riblet tips may also improve the heat transfer characteristics of a riblet surface.

To improve our understanding of the flow physics pertaining to surfaces with widelyspaced riblets, the present study examines widely-spaced riblets spaced 100 and 300 wall-units apart, with a riblet height of 75 wall-units and $l_{g}^{+}$values of 70 and 111, respectively. The friction velocity used for normalizing the quoted geometric dimensions for the smooth and riblet surfaces is the value measured at the streamwise location of the jet in the undisturbed boundary layer over the smooth surface. This riblet height is about five times larger than those considered in published studies reviewed in the foregoing, and corresponds to approximately half of the height of the low-speed streaks observed by Yaras (2007). It is hypothesized that the larger height of the riblets will have a stronger spatial locking effect on the hairpin vortices along the spanwise dimension than was observed by Goldstein \& Tuan (1998) for widely-spaced riblets and therefore will be able to more effectively control the spanwise spacing of the wave packets and hence the extent of their mutual interaction. The height of the riblets in the present study may be larger than that required to achieve this effect and further optimization studies would be needed to reduce the added surface area. Although both riblet surfaces are of the widely-spaced configuration, in the discussions that follow, they will be referred to as the closer-spaced and wider-spaced riblet surfaces. 


\section{Chapter 3}

\section{Experimental Setup and Data Processing}

The experiments were performed in a low-turbulence, open-circuit suction wind-tunnel utilizing a series of $1220 \mathrm{~mm}$ long, $762 \mathrm{~mm}$ wide and $25.4 \mathrm{~mm}$ thick test surfaces installed in a test section of $1830 \mathrm{~mm}$ length, $762 \mathrm{~mm}$ width and $508 \mathrm{~mm}$ height in the configuration shown in Fig. 3.1. The ambient air is drawn into the test section through a contraction of 11.76 area ratio and $2130 \mathrm{~mm}$ length. Downstream of the test section, the air is guided to an axial fan of $775 \mathrm{~mm}$ diameter through a rectangular-to-circular diffusing transition duct $1219 \mathrm{~mm}$ in length with an area ratio of 1.87. A favourable streamwise pressure gradient is imposed on the test surface by orienting the ceiling of the test section at a 13.0-deg. inclination beginning at a height of $318 \mathrm{~mm}$ above the leading edge of the test surface as shown in Fig. 3.1. The flow is then diffused into the transition duct downstream of the test surface with the ceiling oriented at $5.6 \mathrm{deg}$.. The leading edge of each test surface consists of a $50.8 \mathrm{~mm}$ long aluminum section machined to a 15-deg. knife edge from its lower side with an elliptic tip geometry of $15.9 \mathrm{~mm}$ and $3.2 \mathrm{~mm}$ axis dimensions. This leading edge is mounted such that its top surface is flush with the test surface made of medium-density fiberboard. The test surface roughness is less than $30 \mu m$, yielding aerodynamically smooth surface conditions for the flow Reynolds numbers of the present study. The same test-surface configuration was successfully used in an earlier study by the authors' research group (Yaras, 2007).

The first of three test surfaces is smooth and provides the baseline measurement of the turbulent spot. The remaining two test surfaces have streamwise riblets which were shaped with the objective of maximizing passive control of the wave packets prevailing in the turbulent 


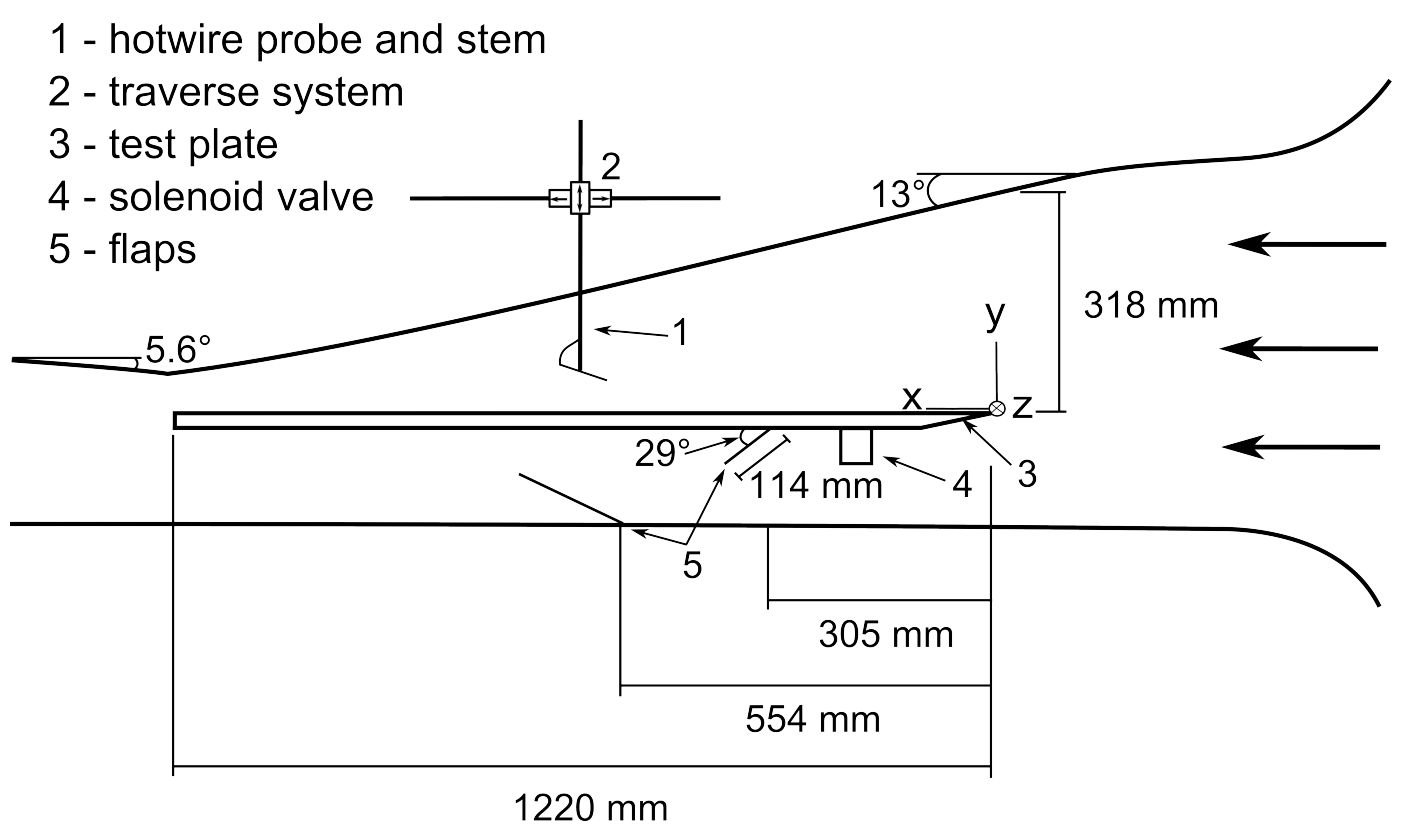

Figure 3.1: Test section configuration

spot. The dimensions and relative positions of wave packets in the turbulent spot for the baseline surface informed the sizing of the riblets. Specifically, spanwise riblet spacings of 100 and 300 wall-units, corresponding to 0.5 and 1.5 times the smooth-surface spanwise spacing of wave packets are considered, with the riblet height set to approximately one-half the height of the low-speed streaks.

No riblets are present on the test surface up to $50.8 \mathrm{~mm}$ from the leading edge. The riblet height increases linearly starting from zero height at $\mathrm{x}=50.8 \mathrm{~mm}$ up to the location of the jet orifice used for spot inception, and remains constant thereafter. The spacing and height of the riblets together with their cross-sectional shape are summarized in Fig. 3.2. The riblets were machined into the test surface such that the jet orifice is centered between two riblets. The trapezoidal riblet geometry is chosen over triangular or rectangular alternatives to produce wider-angled corners in the recesses between riblets and make the tips of the riblets more resistant to damage in a commercial application of such a surface topology. On the surfaces with riblets, the wall-normal (y) coordinate is assigned a value of zero at the base of the riblet.

A turbulent spot is introduced into the laminar boundary layer developing on the test surface by an intermittent jet of air ejected perpendicular to the test surface at a velocity of 


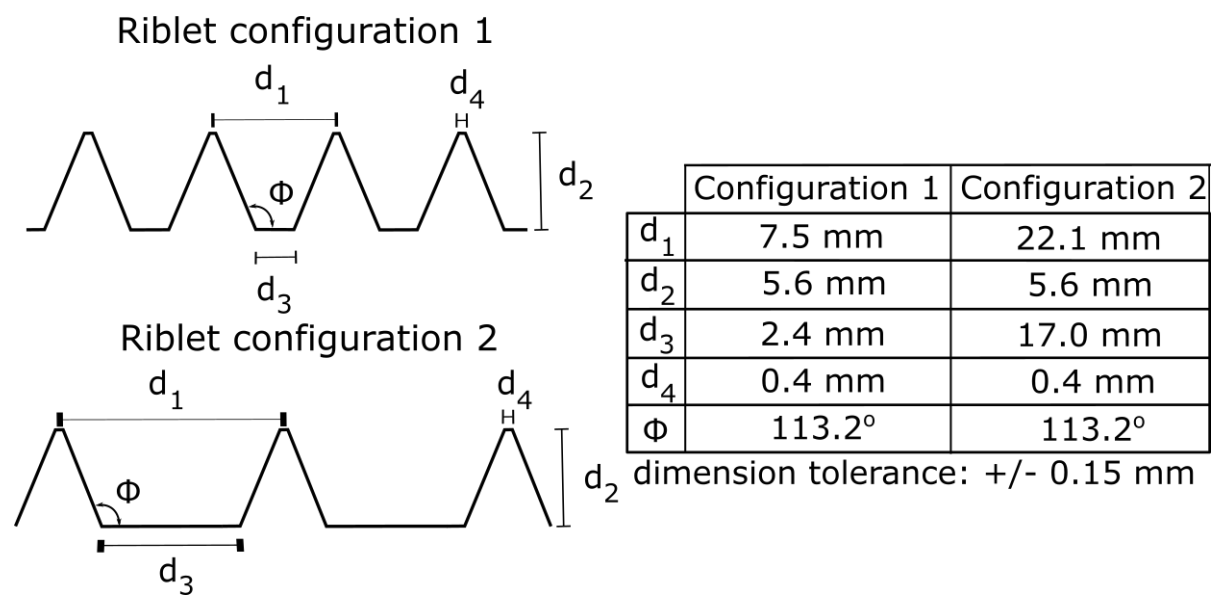

Figure 3.2: Riblet geometry

$60 \mathrm{~m} / \mathrm{s}$ through an orifice of $0.75 \mathrm{~mm}$ diameter located $100 \mathrm{~mm}$ from mid-span and $200 \mathrm{~mm}$ downstream from the test surface leading edge. The jet-to-freestream velocity ratio is 21 and 15 for the low- and high-Reynolds-number cases, respectively. Compressed air supplied to a solenoid valve mounted under the test surface (Fig. 3.1) is used to generate the jet of air. The jet has a duration of $50 \mathrm{~ms}$ and is triggered at a frequency of $1.25 \mathrm{~Hz}$. The time-interval of just under one second between jets was confirmed to be sufficient for the laminar boundary layer developing on the test surface to fully recover between sequential turbulent spots.

Since the streamwise flow blockage due to the solenoid valve under the test plate is localized, a deflector plate of the same height as the solenoid valve is mounted to the test plate just downstream of the valve to produce a spanwise-uniform flow blockage. A second flap mounted on the test-section floor (Fig. 3.1) is set to an angle such that the leading-edge stagnation point is slightly biased towards the measurement side of the test plate, thus eliminating the potential of local flow separation on this side of the test plate. Through measurements conducted with a conical four-hole pressure probe of 128 degree angle and $19 \mathrm{~mm}$ base diameter positioned $50 \mathrm{~mm}$ upstream of and vertically aligned with the test surface leading edge, the flow incidence was confirmed to be spanwise-uniform at a value of $1.3 \pm 0.5$ degrees relative to the test surface.

The streamwise velocity component in the boundary layer and in the freestream was measured using a single-sensor hotwire probe with a tungsten sensor of $1.3 \mathrm{~mm}$ length and 
$5 \mu \mathrm{m}$ diameter mounted on a probe stem $165 \mathrm{~mm}$ in length and controlled by an AN-1003 constant-temperature anemometer at an overheat ratio of 1.76 and a frequency response of $12 \mathrm{kHz}$. A TSI Air Velocity Calibrator with an air jet of 10-mm diameter discharging from a converging nozzle into the surroundings was used to calibrate the probe based on the measurement of 20 jet velocities evenly distributed over a range of 0 to $12 \mathrm{~m} / \mathrm{s}$ with the probe stem aligned with the flow. The measurement uncertainty in the velocity based on this calibration procedure and a 9th-order polynomial fit to the calibration data was determined to be $\pm 2 \%$, except for velocities below $0.5 \mathrm{~m} / \mathrm{s}$ which had an uncertainty of $\pm 5 \%$ at a $99 \%$ confidence interval. The anemometer output voltage was corrected to account for any changes in ambient temperature of up to $4.0^{\circ} \mathrm{C}$ during experiments (Bruun, 1995). The axis of the probe stem was inclined at an angle of 10 degrees to the test surface to mitigate any blockage effects in the near-wall region (Fig. 3.1). This orientation is consistent with earlier studies (Roberts \& Yaras, 2004; Yaras, 2007) and was established through a sensitivity study to minimize the blockage of the measured flow region by the probe stem. A bias error of less than $1 \%$ resulted from the differences in the flow alignment of the probe between the calibration and measurement setups. In near-wall measurements, the loss of heat from the probe sensor to the surface can cause significant bias errors which are primarily affected by the thickness and thermal conductivity of the test surface material. This effect is generally limited to a distance of $y^{+}<3.5$ from the wall (Bruun, 1995; Durst \& Zanoun, 2002). The use of a poorly-conducting surface $(k=0.3 \mathrm{~W} / \mathrm{mK})$ further mitigates the heat transfer effect such that a benchmark test measurement of the Blasius boundary layer in the present study revealed negligible deviation from the expected velocity profile.

Measurements were performed $250 \mathrm{~mm}, 400 \mathrm{~mm}$, and $500 \mathrm{~mm}$ downstream of the location of the jet. At each of the streamwise locations, the hotwire probe was traversed through 80 positions normal to the test surface at multiple spanwise locations. For measurements on the surfaces with streamwise riblets, 21 additional wall-normal positions were required to measure the velocity within the grooves. The measurement positions were clustered near the surface to capture the higher prevailing velocity gradients, and extended past the height of the turbulent spot to $15 \mathrm{~mm}$ above the test surface. The smallest spacing between consecutive 
wall-normal positions was set to $0.1 \mathrm{~mm}$ and corresponds to the regions where preliminary measurements indicated the presence of streaky structures in the streamwise velocity field. The wall-normal traverses were performed at 21 spanwise positions between $\mathrm{z}=-7.5 \mathrm{~mm}$ and $\mathrm{z}=67.5 \mathrm{~mm}$ at $3.75 \mathrm{~mm}$ increments capturing just over the half-width of the turbulent spot up to the spanwise position where the flow is undisturbed by the spot. Additionally, measurements with a spanwise spacing of $1.25 \mathrm{~mm}$ were performed to capture the streaky structures with higher resolution. The refined measurements captured a single low-speed streak which required up to 13 additional wall-normal traverses. Wall-normal traverses were performed using a motorized traverse system with an absolute positioning uncertainty of $\pm 0.1 \mathrm{~mm}$ and a relative positioning uncertainty of $\pm 0.025 \mathrm{~mm}$.

At each measurement position, the anemometer output was recorded through 75 spotgeneration cycles, which was confirmed to be sufficient for statistical convergence based on a sensitivity analysis of the ensemble-averaged turbulence kinetic energy at several locations within the spot. The signal was sampled at 10,240 Hz which was deemed sufficient to resolve the highest frequency fluctuations with significant power spectral density in the flow as per the Nyquist criterion. The data acquisition was initiated simultaneously with the triggering of the jet and was terminated $0.4 \mathrm{~s}$ later which corresponded to 4096 samples. This duration was sufficient to track the turbulent spot at the measurement plane farthest along the test surface. The quantization error of the data acquisition system was insignificant in comparison to the aforementioned uncertainty associated with the calibration of the hotwire sensor and any errors caused by wall proximity.

The structure of the turbulent spot was studied through the ensemble-averaged perturbation component of the measured velocity:

$$
\tilde{u}(x, y, z, t)=\frac{\hat{u}(x, y, z, t)-u_{l}(x, y, z)}{u_{e}}
$$

where $\hat{u}$ is the ensemble average of the measured velocity, $u_{e}$ is the local undisturbed boundarylayer edge velocity and $u_{l}$ is the local undisturbed (laminar) velocity obtained by timeaveraging the velocity signal from the moment the turbulent spot is triggered until it reaches 
the streamwise location of measurement. While the orientation of the hotwire probe makes it sensitive to both the streamwise and wall-normal velocity components, the wall-normal component is an order of magnitude less than the streamwise component in the measured region of flow. Therefore the velocity measured by the hotwire will be denoted by the streamwise component, $u$, to reflect the dominant velocity component.

In the study of the measurement results, the perturbation velocity was complemented by the root-mean-square of the fluctuations in the measured velocity, $u^{\prime}(x, y, z, t)$. This was determined using the following expression:

$$
u^{\prime}(x, y, z, t)=\sqrt{\frac{\sum_{n} \frac{u(x, y, z, t)-\hat{u}(x, y, z, t)^{2}}{u_{e}^{2}}}{n}}
$$

where $\mathrm{n}$ is the number of measurement cycles included in the ensemble average.

The spanwise component of vorticity in the undisturbed boundary layer was determined by fitting a ninth-order polynomial to the wall-normal velocity profile, and determining the value of $\partial u / \partial y$ based on this polynomial. This method reduces the effect of scatter in the velocity measurements on the calculated vorticity to enable easier interpretation of the spatial distribution of vorticity. The streamwise gradient of the wall-normal component of velocity, $\partial v / \partial x$, is neglected in the calculation of this vorticity component based on order-of-magnitude comparison of $\partial v / \partial x$ and $\partial u / \partial y$.

To quantify the growth rate of the turbulent spot, the propagation parameter, $\sigma$, was determined using the following equation proposed by Narasimha (1985) for both favourable and mild adverse pressure gradients provided that the pressure gradient does not vary significantly within the transition region:

$$
\sigma=u_{e}\left(u_{T E}^{-1}-u_{L E}^{-1}\right) \tan (\alpha)
$$

where $u_{e}$ is the local boundary layer edge velocity, $u_{T E}$ and $u_{L E}$ are respectively the leading and trailing edge celerities of the spot, $\alpha$ is the spreading half-angle in the streamwise-spanwise plane measured from the location of spot inception, and $\tan (\alpha)$ quantifies the lateral growth rate of the spot. The spot propagation parameter represents the rate of longitudinal growth 
of the spot which can be calculated through $\sigma /[\tan (\alpha)]$. The measured values of the spot propagation parameter were compared to the values predicted by the empirical correlation developed by Gostelow et al. (1996). This correlation was developed for flows under pressure gradients characterized by values in the range of -0.6 to 0.6 for Thwaites' pressure gradient parameter, $\lambda_{\theta}=\left(\theta^{2} / \nu\right) d u_{e} / d x$, where $\theta$ is the local momentum thickness of the undisturbed boundary layer and $\nu$ is the kinematic viscosity:

$$
\sigma=0.03+0.37 /\left(0.48+3.0 e^{52.9 \lambda_{\theta}}\right)
$$




\section{Chapter 4}

\section{Operating Conditions and Undisturbed Boundary Layer Development}

Experiments were conducted at Reynolds number values of $2.2 \times 10^{5}$ and $3.1 \times 10^{5}$ based on the length of the test surface and freestream velocity values of $2.8 \mathrm{~m} / \mathrm{s}$ and $4.0 \mathrm{~m} / \mathrm{s}$, respectively, with ambient air as the working fluid. The freestream velocity was measured $25.4 \mathrm{~mm}$ downstream of the leading edge and $25.4 \mathrm{~mm}$ above the test surface at the spanwise location of spot inception, and was correlated to the contraction pressure difference of the wind tunnel before commencing with the measurements of the turbulent spot. The Reynolds number was kept constant to within $\pm 0.7 \%$ during the measurements. The freestream turbulence intensity measured at the reference location was $0.4 \% \pm 0.1 \%$ in all cases, and was found to be uniform throughout the test section. The uniformity of the uniaxial flow entering the test section was such that the flow velocity remained within $2.0 \%$ of the centerline value at all locations outside the test-section boundary layers.

The inclination of the test section ceiling resulted in freestream acceleration parameter values of $\eta=\nu / u_{e}^{2} d u_{e} / d x=4.0 \times 10^{-6}$ and $2.9 \times 10^{-6}$ for the low and high Reynolds number values, respectively. This level of acceleration is within the range defined by Jones \& Launder (1972) to cause re-laminarizing of a turbulent boundary layer. The relatively high streamwise acceleration rate was chosen to reduce the extent of spanwise meandering of the streaky structures appearing in the velocity field of the turbulent spot, enabling more precise investigation of their structures through ensemble-averaged data. 
In the presentation of the results, length dimensions are normalized by the undisturbed boundary-layer displacement thickness, $\delta_{r e f}^{*}$, at the streamwise location of the jet. The nondimensional time, $\tau$, is calculated as $\tau=t\left(u_{r e f} / \delta_{r e f}^{*}\right)$ where $u_{r e f}$ is the edge-velocity of the undisturbed boundary layer at the streamwise location of the jet, $t$ represents time, and $\mathrm{t}=0 \mathrm{~s}$ corresponds to the instant the jet is triggered.

The favourable streamwise pressure gradient ensured that the undisturbed boundary layer remained laminar. Figure 4.1 shows the streamwise velocity profiles for this boundary layer at $\mathrm{x}=600 \mathrm{~mm}$. The streamwise velocity profile over the smooth surface (Fig. $4.1 \mathrm{a}, \mathrm{b})$ is consistent with the Falkner-Skan self-similar boundary-layer solution defined by a value of 0.6 for the $\beta$ parameter. Comparing the profiles in Fig. 4.1 c, e to Fig. 4.1 a and Fig. $4.1 \mathrm{~d}$, f to Fig. $4.1 \mathrm{~b}$, the riblet effect on the wall-normal profile of streamwise velocity is observed to be highly dependent on the proximity to the nearest riblet. On the surface with closer-spaced riblets (Fig. $4.1 \mathrm{c}, \mathrm{d}$ ), the wall-normal gradient of velocity at the riblet tip is notably higher than for the smooth surface, with the velocity measured $0.13 \delta_{\text {ref }}^{*}$ above the riblet tip having a value of $u / u_{e}=0.8$ compared to the value of 0.4 at the same distance from the surface for the smooth-surface case. This is explained by the spanwise-limited effect of the surface drag of the narrow flat tip of the riblet, which enables the fluid immediately above the riblet tip to maintain higher streamwise momentum as it is energized by the spanwise-adjacent higher-speed fluid at the same wall-normal location. In contrast, within the groove formed by adjacent riblets, the combined effect of surface drag along the side walls and base of the groove yields lower streamwise momentum below the riblet tip. As a result, an inflection point is observed in the velocity profiles at spanwise locations that fall within the groove. As the riblet spacing is increased, the effect of the surface drag on the side walls of the riblets on the velocity profile at the center of the groove would be expected to diminish. This is indeed observed to be the case when the velocity profile at the center of the groove on the test surface with wider-spaced riblets (Fig. $4.1 \mathrm{e}, \mathrm{f}$ ) is compared to the corresponding profile with closer-spaced riblets (Fig. $4.1 \mathrm{c}, \mathrm{d}$ ). The flow at the groove centerline of the wider-spaced riblet surface (Fig. 4.1 e, f) still has notably larger momentum deficit than on the smooth 
surface (Fig. $4.1 \mathrm{a}, \mathrm{b}$ ), indicating that the influence of the surface drag of the riblet side walls extends across the full width of the groove even for the wider-spaced riblet surface.
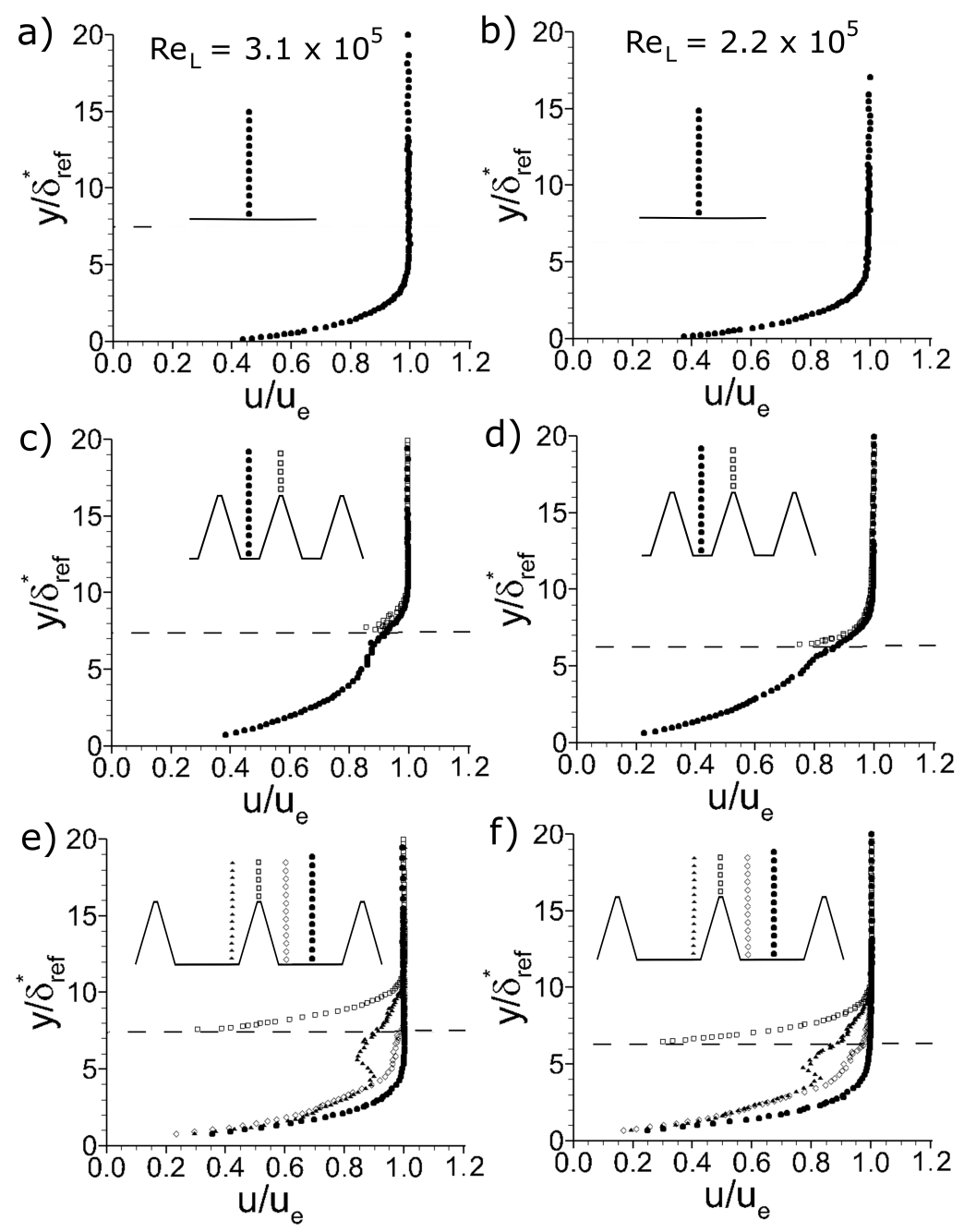

Figure 4.1: Undisturbed boundary-layer velocity profiles for $R e_{L}=3.1 \times 10^{5}$ (a,c,e) and $R e_{L}=2.2 \times 10^{5}(\mathrm{~b}, \mathrm{~d}, \mathrm{f})$. For the riblet surfaces, the dashed horizontal line identifies the location of the riblet tip.

Within the groove of the wider-spaced riblet surface, the velocity profile closer to the riblet is observed to develop an inflectional shape, similar to the velocity profile in the groove of the closer-spaced-riblet surface. Interestingly, this inflection is noted to be very strong on one side of the riblet and nearly absent on the other. This difference is caused by a small $(0.3 \pm 0.1$ degree $)$ yaw misalignment of the freestream relative to the riblets of this surface. 
Such a flow misalignment causes a wake region on the downwind side of the riblet, prompting the development of the strong inflectional shape of the velocity profile. In contrast, the windward side of the riblet benefits from a local flow acceleration that reduces the magnitude of the inflection in the velocity profile. The yaw misalignment is observed to also affect the velocity profile on the riblet tip, with the profile having a notably larger amount of momentum deficit compared to the corresponding profile of the closer-spaced-riblet surface. Similar freestream yaw misalignments with the riblets was observed not to affect the velocity profiles on the closer-spaced-riblet surface, likely because the closer proximity of the riblets enhances the shielding effect of a riblet on its spanwise downwind neighbour. While the stated flow misalignment was not intentional, it is quite informative in illustrating the sensitivity of the undisturbed boundary layer development on the riblet surface to yaw misalignment, and the effect of riblet spacing on this sensitivity. 


\section{Chapter 5}

\section{Results and Discussion}

\subsection{Turbulent spot development over a smooth surface}

This discussion is intended to provide a context for the ensuing presentation of the turbulent-spot development over surfaces with riblets. The turbulent spot at both Reynolds

numbers is visualized in Fig. 5.1 in the z-t plane through the perturbation velocities at a wall-normal distance which most clearly shows the streak structures of the spot. In the interest of clarity, the axes of this figure are not shown to scale. The perimeter of the turbulent spot is denoted by a contour of the rms fluctuation in the velocity magnitude. The illustrated rms value is one which most clearly shows the outline of the spot. The turbulent spot is observed to form an arrowhead shape pointing in the upstream direction. This orientation, while less common than the downstream-pointing arrowhead shape, has been observed in previous research where the spot is formed through the bypass mode of transition triggered by a disturbance that is dominant in the outer region of the boundary layer (e.g. Wu et al., 1999; Zhong et al., 1998; Yaras, 2007). In the present experiments, the spot is triggered by a strong transverse jet similar to that used by Yaras (2007) which reaches far into the freestream. The interaction between the jet and the main flow creates a local adverse pressure gradient at the wall on the downstream side of the jet, which results in local separation of the main-flow boundary layer. The jet entrains and re-orients the vorticity of the separated boundary layer to produce coherent vortical structures in its wake (Fric \& Roshko, 1994). These vortical structures quickly overtake any other transient flow structures that may develop through the 
interaction of the jet with the inner region of the main-flow boundary layer and are convected above a region of undisturbed laminar flow. Through the shear-sheltering mechanism, only the lower-frequency fluctuations are able to penetrate the inner region of the boundary layer, where they form pre-transitional streak structures in the streamwise velocity field, otherwise known as Klebanoff modes (Jacobs \& Durbin, 2001). Bypass transition is initiated when one or more of these inviscid-unstable low-speed streaks is perturbed by the vortical structures in the outer region of the boundary layer (Brinkerhoff \& Yaras, 2015; Hack \& Zaki, 2014). These perturbations incite a secondary instability in the low-speed streak, leading to the simultaneous formation of several hairpin vortices along its streamwise extent. This flow development, in turn, initiates similar dynamics in nearby Klebanoff-mode streaks, thus facilitating rapid spanwise spreading of the resultant turbulence. This spreading mechanism achieves the spanwise extent of the turbulent spot illustrated in Fig. 5.1 over a streamwise distance of less than $75 \mathrm{~mm}$ (approximately $100 \delta_{\text {ref }}^{*}$ ) downstream of the jet. It is worth noting that, owing to the strongly favourable streamwise pressure gradient imposed along the length of the test surface, the spot undergoes very little spanwise growth downstream of the region of initial spot formation.

The interaction of the jet with the main-flow boundary layer is observed to be sensitive to Reynolds number as suggested by the size of the turbulent spot originating from this interaction for the two values of the Reynolds number. However, the number, size, and perturbation-velocity magnitude of the streaky structures remain unchanged within this range of Reynolds-number values. The spot contains four low-speed streaks with spanwise spacing of $211 \pm 22$ and $206 \pm 17$ wall-units for $R e_{L}=2.2 \times 10^{5}$ and $R e_{L}=3.1 \times 10^{5}$, respectively. This spanwise spacing is consistent with published studies (Jones \& Launder, 1972; Yaras, 2007), which have shown that the average streak spacing in turbulent boundary layers under a streamwise pressure gradient that promotes relaminarization is two to three times larger than the streak spacing in zero-pressure-gradient boundary layers. The magnitude of the perturbation velocity of the inboard low-speed streaks of the spot is notably lower than that of the outermost streak. This variation in streak strength is observed more clearly through 

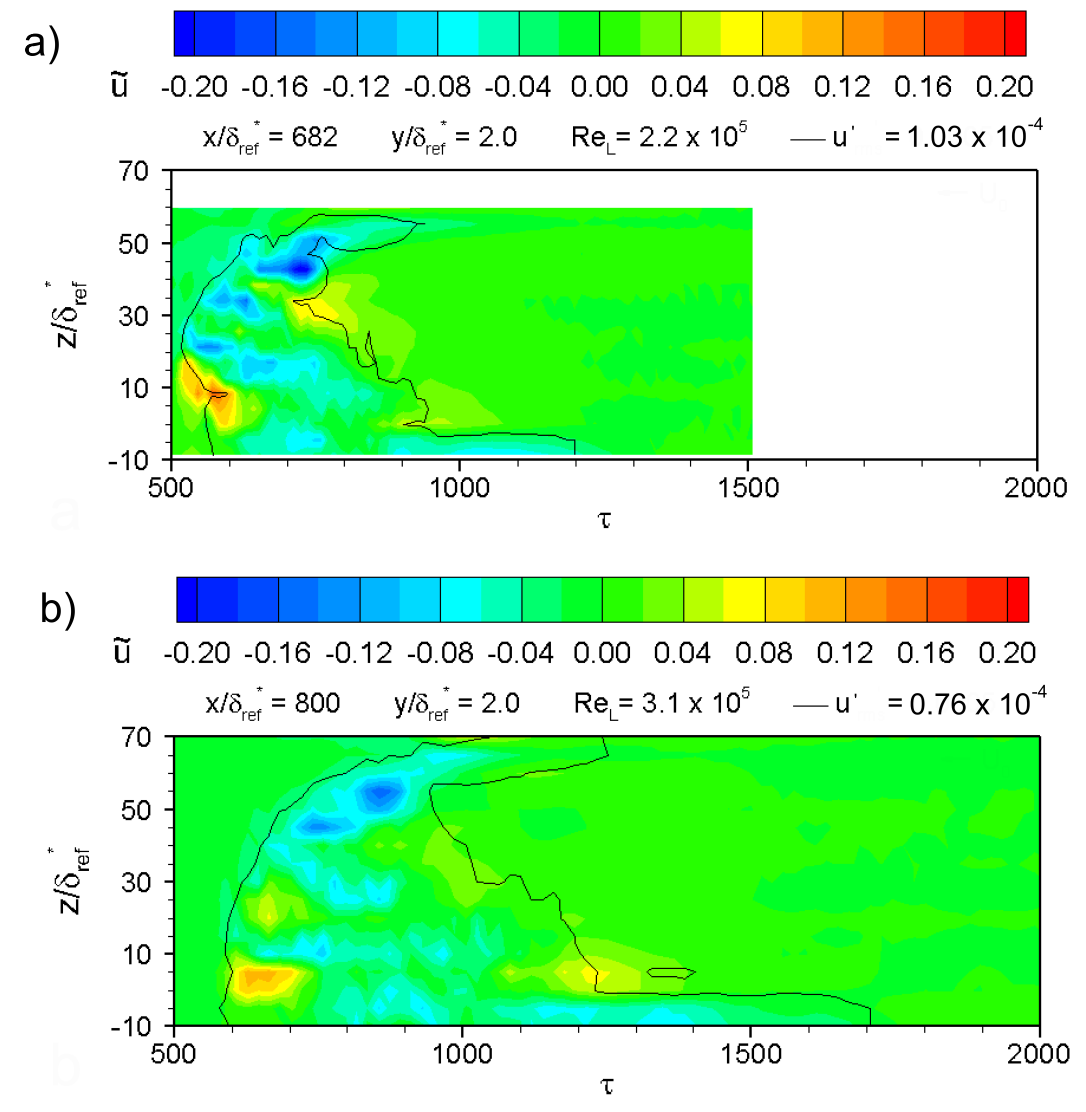

Figure 5.1: Perturbation velocity in the z-t plane on the smooth surface measured at $\mathrm{x}=600 \mathrm{~mm}$ for a) $R e_{L}=2.2 \times 10^{5}$ and b) $R e_{L}=3.1 \times 10^{5}$

the perturbation velocity distribution in the y-z plane depicted in Fig. 5.2, where each illustrated plane is approximately at the same location relative to the leading and trailing edges of the spot. The relative weakness and spanwise-smeared appearance of the inboard streaks is suggestive of greater spanwise meandering of these streaks compared to their outboard counterparts. The magnitude of the velocity deficit within the streaks reaches approximately $20 \%$ $\pm 1.0 \%$ of the local freestream velocity. This is below the threshold of $26 \%$ found by Elofsson et al. (1999) and Brandt \& Henningson (2002) to be necessary for sinuous streak instability to occur in zero-pressure-gradient boundary layers. Additionally, the strong favourable pressure gradient of the present study further promotes the stability of the streaky structures. In the absence of streak instability promoting meandering of the streaks, the observed results for 
the inboard streaks may be caused by the mutual interaction of spanwise-adjacent wave packets. Such interaction is reduced for the outboard streaks given the undisturbed conditions prevailing beyond the perimeter of the turbulent spot.
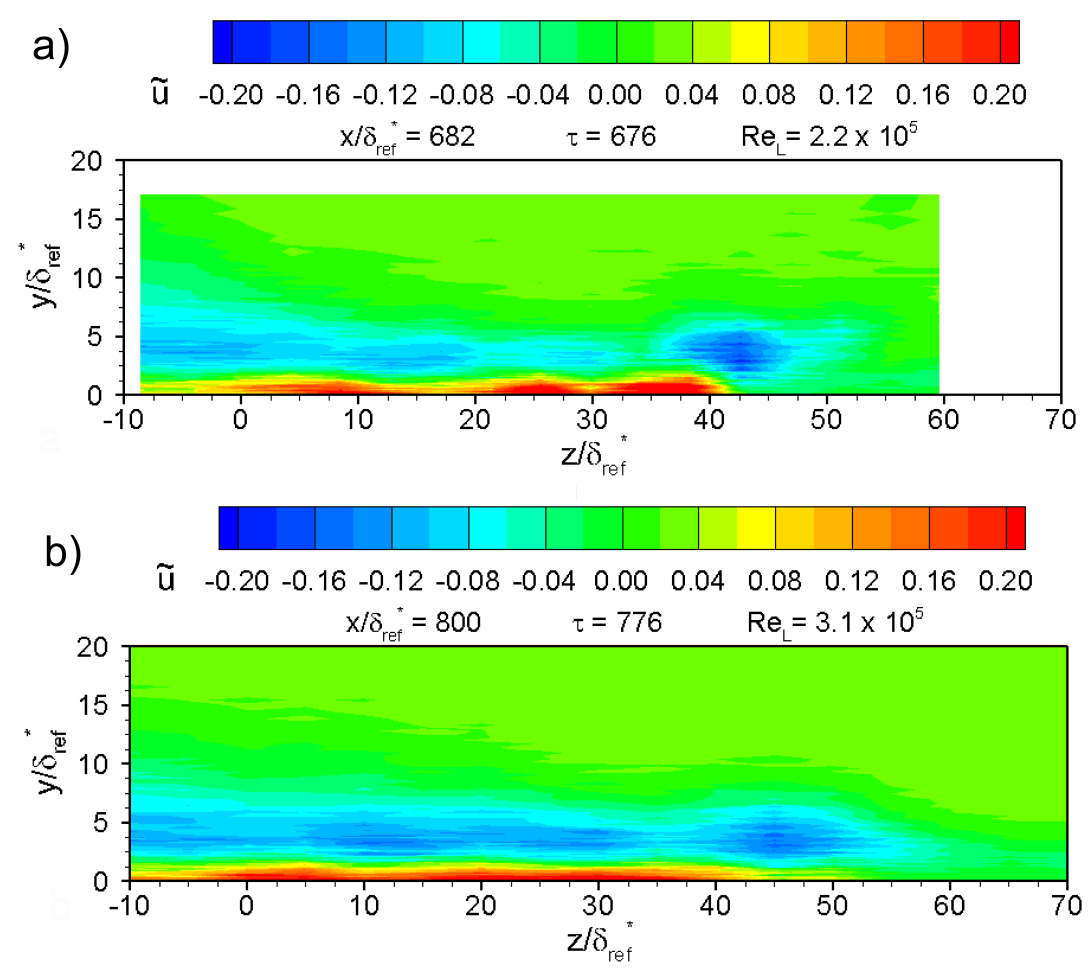

Figure 5.2: Perturbation velocities in the $y-z$ plane on the smooth surface measured at $\mathrm{x}=600 \mathrm{~mm}$ for a) $R e_{L}=2.2 \times 10^{5}$ and b) $R e_{L}=3.1 \times 10^{5}$

The overhang and calmed regions, which are typical features observed respectively at the leading edge and in the wake of a turbulent spot, are visible through observation of the perturbation velocity field in the y-t plane as shown in Fig. 5.3 for $R e_{L}=3.1 \times 10^{5}$. The overhang region at the spot leading edge consists of low-speed fluid that is convected away from the wall by the heads of the older hairpin vortices. The larger distance of these older structures from the wall enables them to convect faster in the streamwise direction, resulting in the overhang pattern over a region of undisturbed flow. For example, the presence of a leading-edge overhang is readily apparent at $z / \delta_{\text {ref }}^{*}=20.0$, which coincides with one of the low-speed streaks of the spot. The wake region of the spot begins downstream of the trailing edge hairpin vortices at approximately $\tau=1000$ in Fig. 5.3. The calmed region is known 
to have greater stability promoted by high-speed streaks which yield relatively fuller velocity profiles. Brinkerhoff \& Yaras (2014) explain these streaks to be the result of the downwash created by streamwise aligned sheets of enhanced spanwise vorticity that extend from the spot into the wake. These sheets are embedded in the slow-moving fluid near the surface and produce a calmed region that grows longitudinally along with the spot. The length of these sheets are limited by viscous diffusion which results in a calmed region of finite streamwise extent. The length of the calmed region is also sensitive to streamwise pressure gradient, with a favourable pressure-gradient suppressing the streaks to produce a smaller calmed region (Katz et al., 1990). The calmed region in the present study is comparable in size to other studies with similar pressure gradients (Yaras, 2007) and does not vary within the range of Reynold-number values considered.

The streamwise and spanwise extents of the spot are measured at each measurement plane through the spatial distribution of the rms value of the fluctuating component of the measured velocity, $u^{\prime}$. The spot dimensions corresponding to 14 values of $u^{\prime}$ equally spaced between $1.0 \times 10^{-4}$ and $24.3 \times 10^{-4}$ were averaged to yield one streamwise and one spanwise dimension at each streamwise measurement location. These dimensions allowed the spreading angle and the leading- and trailing-edge celerities to be calculated. The streamwise variation of the propagation parameter of the turbulent spot determined using these spot parameters as per Eq. 3.3 is shown in Fig. 5.4 with an uncertainty of $\pm 4.0 \%$. Despite the favourable pressure gradient, the spot initially propagates very near the rate predicted by Eq. 3.4 for a zero-pressure-gradient boundary layer which is shown in Fig. 5.4 along with the expected propagation parameter of a spot in a boundary layer with the favourable pressure gradient of the present study. As the spot convects downstream, its propagation parameter gradually adjusts towards the value expected under the current pressure gradient. This is likely caused by the streamwise pressure gradient in close vicinity of the jet being dictated dominantly by the wake dynamics (Brinkerhoff \& Yaras, 2012). A similar trend is noted in streamwise variation of the spreading angle of the spot. 

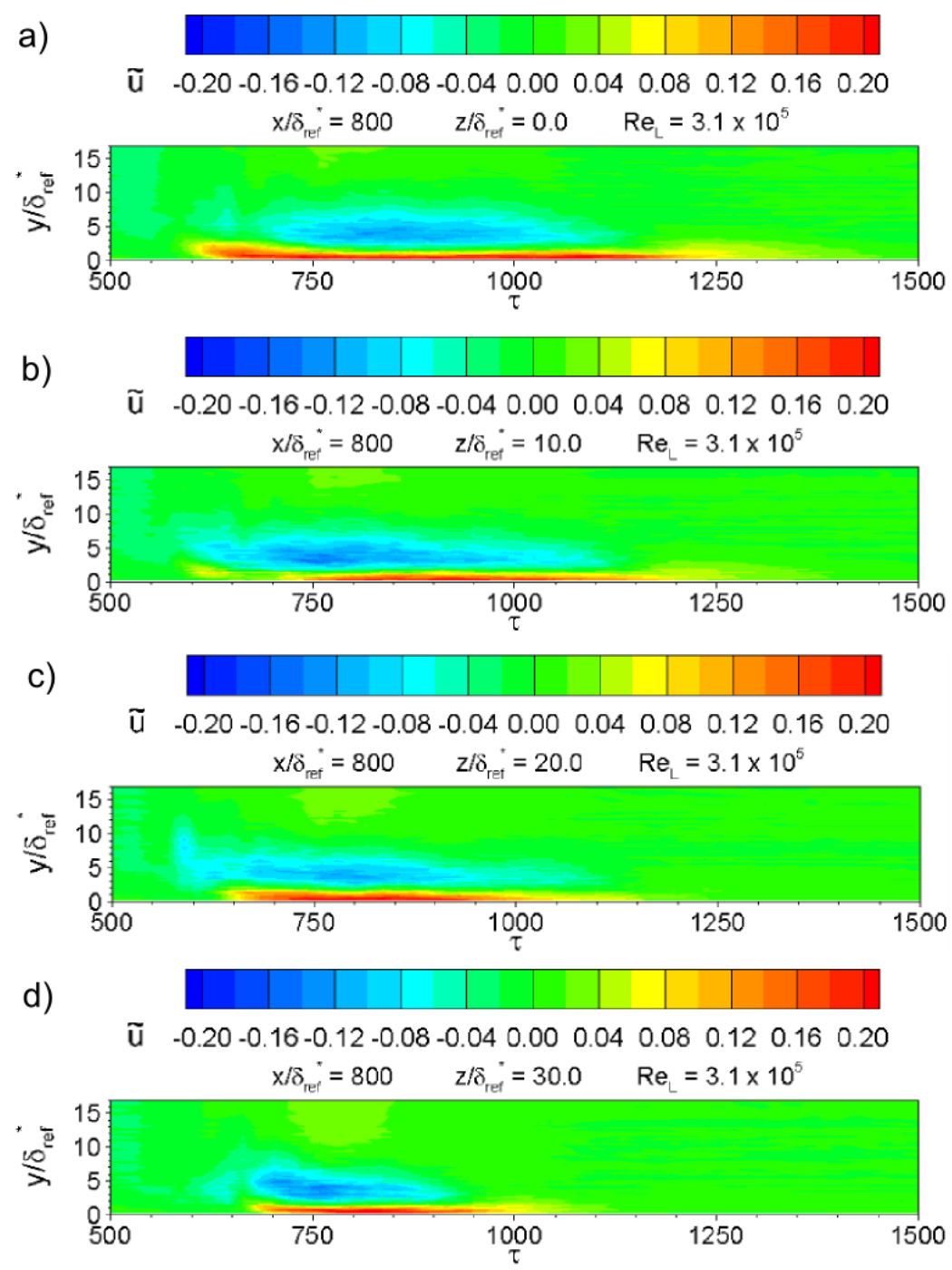

Figure 5.3: Perturbation velocity distribution in the y-t plane over the smooth surface measured at $\mathrm{x}=600 \mathrm{~mm}$ at a) $\left.\left.z / \delta_{\text {ref }}^{*}=0.0, \mathrm{~b}\right) z / \delta_{\text {ref }}^{*}=10.0, \mathrm{c}\right) z / \delta_{\text {ref }}^{*}=20.0$, and d) $z / \delta_{\text {ref }}^{*}=30.0$

\subsection{Effects of riblets on the coherent structures of turbulence}

The spanwise vorticity of the undisturbed boundary layer is the primary source of vorticity for the hairpin vortices of the turbulent spot. Accordingly, any mechanism that influences the spatial distribution of vorticity in the boundary layer has the potential to affect the development of the hairpin vortices and their spatial organization. Fig. 5.5 shows the wall-normal 


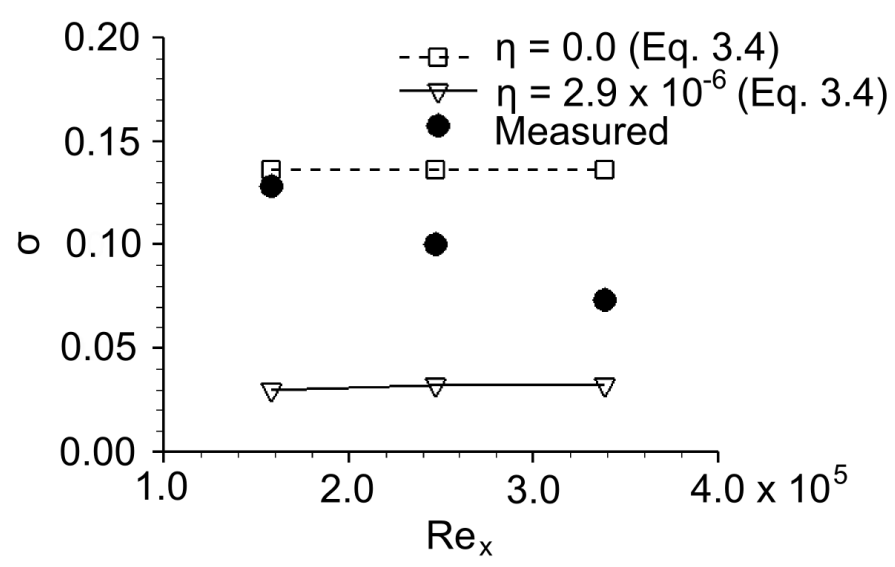

Figure 5.4: Variation of the spot propagation parameter for $R e_{L}=3.1 \times 10^{5}$ over the smooth surface

distribution of the time-averaged streamwise velocity and the corresponding spanwise component of vorticity in the absence of the turbulent spot. The effect of the riblets on the undisturbed boundary layer was observed to be the same for both values of measured flow Reynolds number; for brevity, only the results for $R e_{L}=3.1 \times 10^{5}$ are presented. The spanwise-oriented vorticity lines in the boundary layer are expected to deform in the streamwise-spanwise plane owing to the spanwise non-uniformity of streamwise velocity prompted by the presence of the riblets. Such re-orientation of the vorticity vector would promote the formation of hairpin vortices by adding a streamwise component to the background vorticity and promoting spanwise-periodic waviness of the vorticity lines akin, for example, to the process that occurs in the later stages of instability in natural transition of boundary layers through the Tollmien-Schlichting viscous instability mechanism. The effect of the riblets on the spanwise vorticity distribution can be readily observed in Fig. 5.5. For example, in Fig. 5.5a, a region of non-zero spanwise vorticity exists at the wall-normal position of the inflection point. The corresponding wall-normal location over the smooth and wider-spaced riblet surface (Fig. $5.5 \mathrm{c}$ ) has zero vorticity.

The effect of the altered undisturbed vorticity field over the riblet surfaces on the coherent structures in the spot can be observed in Fig. 5.6 which shows the perturbation velocity field for $R e_{L}=3.1 \times 10^{5}$ for all three test surfaces. The low-speed streaks over the riblet surfaces 

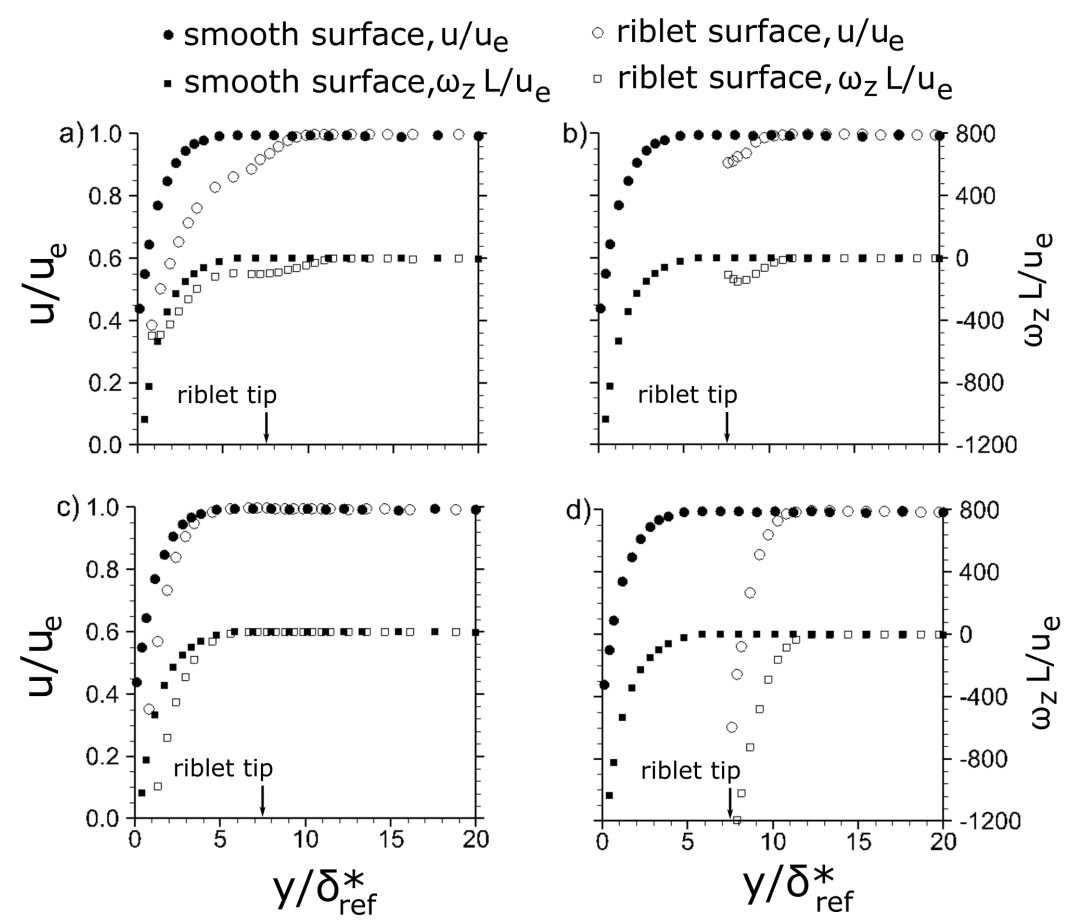

Figure 5.5: Comparison of the ensemble-averaged streamwise velocity and spanwise vorticity at $\mathrm{x}=600 \mathrm{~mm}$ and $R e_{L}=3.1 \times 10^{5}$ between the smooth surface and the a) closer-spaced riblet; groove center b) closer-spaced riblet; riblet tip c) wider-spaced riblet; groove center d) wider-spaced riblet; riblet tip

exhibit a tendency of locking onto the position of the riblet tips where they likely reside as depicted schematically in Fig. 5.7. The present riblets with a height of 75 wall-units and spanwise spacings of 100 and 300 wall-units have a significantly greater influence on the spanwise location of the wave packets than was observed by Goldstein \& Tuan (1998) over riblets with a height of 10 wall-units and spanwise spacings of 65.3 and 375 wall-units.

The larger distance between adjacent low-speed streaks on the surface with the widerspaced riblets reduces the opportunity for mutual spanwise interaction between neighbouring wave packets and, along with the reduced meandering of the streak as a result of the locking effect of the riblet, contributes to the more coherent and stronger appearance of the streaks (Fig. 5.6c) relative to those on the smooth surface. The wider-spaced riblets may also be able to reduce the population of wave packets per unit spanwise distance compared to the smooth-surface baseline case, thus altering the extent of wall-normal turbulent mixing per unit 
a)
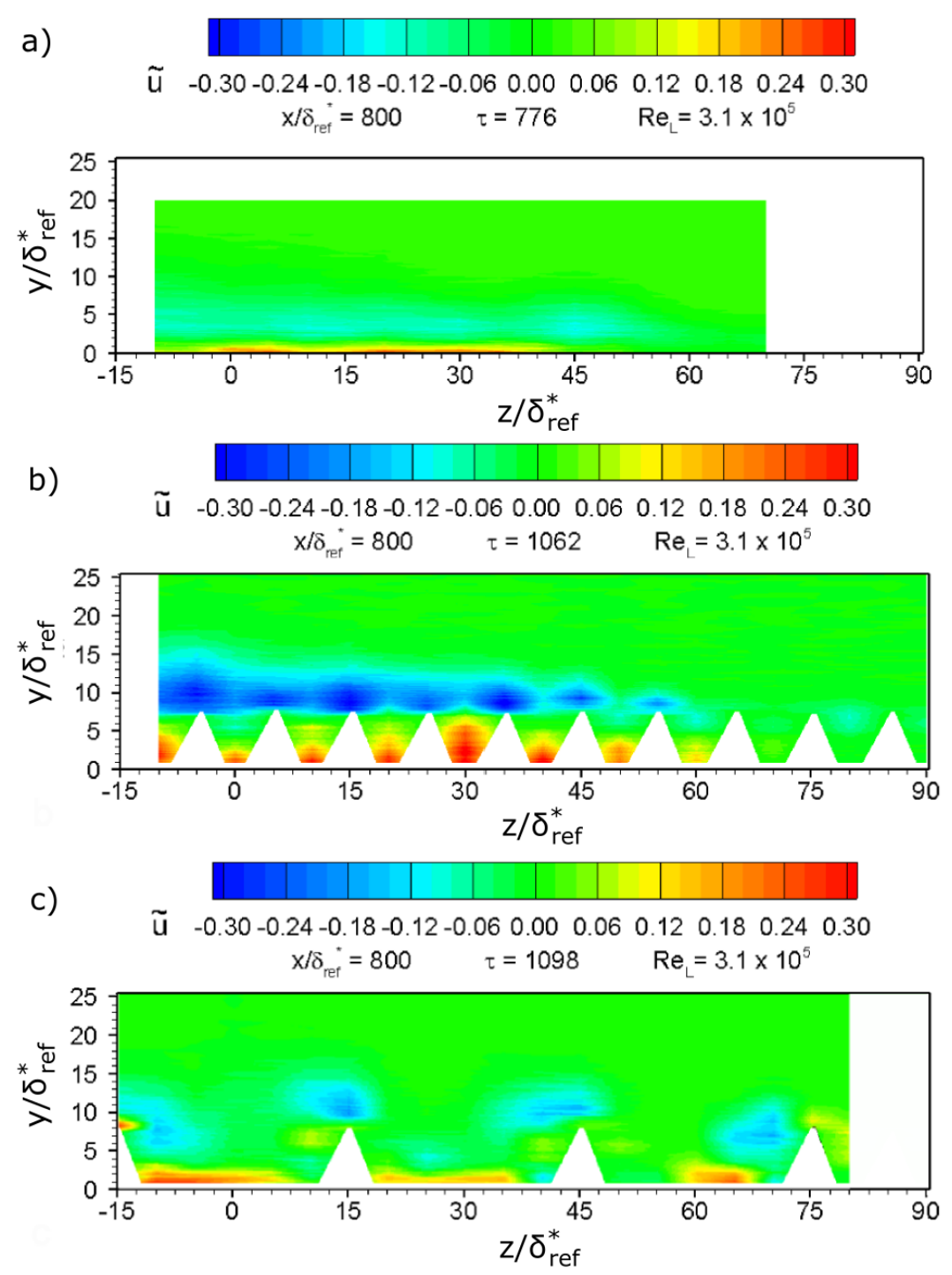

Figure 5.6: Perturbation velocity distribution in the y-z plane a) smooth surface b) surface with closer-spaced riblets c) surface with wider-spaced riblets

surface area. Comparison of the results shown in Figs. 5.6a and c, for example, indicate one fewer low-speed streak for the riblet surface with wider spacing than for the smooth surface over the same spanwise distance. Of course, as the riblet spacing is increased, a threshold would eventually be reached beyond which hairpin vortices may form within the grooves through the previously-described mechanism identified over smooth surfaces, in addition to those associated with the riblet tips. The streamwise velocity profile at the center of the groove for the wider-spaced riblet approaches that measured on the smooth surface 


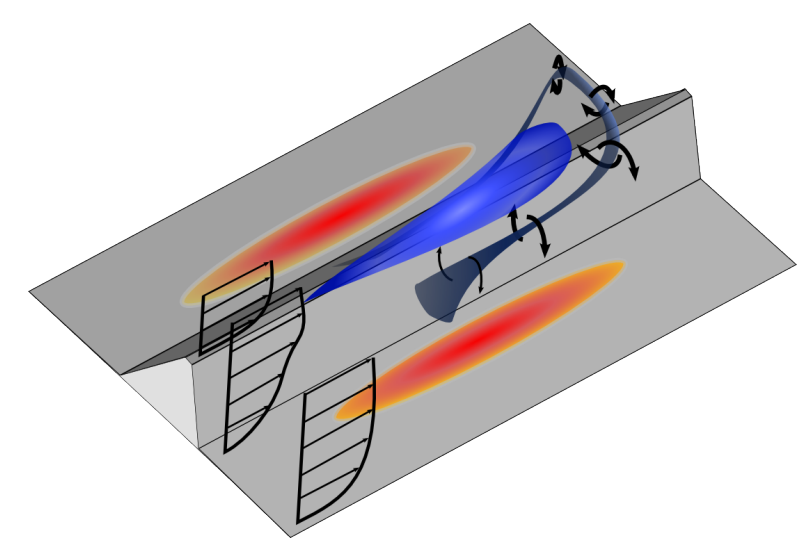

Figure 5.7: Schematic of a hairpin vortex and its induced motions over an isolated riblet. The red and blue regions denote regions of positive and negative perturbation velocities, respectively

(Fig. 5.5c). Accordingly, the wider-spaced riblet surface considered here may represent a spacing that is not too far from the suggested threshold.

The topology of the spot over the wider-spaced riblet surface is further visualized through the fluctuation and perturbation velocities in z-t planes above the riblet tip (Fig. 5.8a) and below the riblet tip (Fig. 5.8b). The reduced spanwise meandering of the streaks compared to the smooth-surface case (Fig. 5.1) is evident. The single low-speed streak which forms the tip of the arrowhead shape of the spot is seen to reside within the groove. The lack of low-speed fluid over the riblets adjacent to this streak may suggest that this streak is associated with the initial Klebanoff-mode. Development of this streak through secondary instabilities leads to the formation of neighbouring streaks. The spanwise positions of these streaks and associated wave packets are observed to be biased towards the riblet tips.

The turbulent spot over the closer-spaced riblet surface is visualized through the fluctuation and perturbation velocities in the z-t plane in Fig. 5.9. The spot over this surface is noted to be larger in both the streamwise and spanwise dimensions than the spots over the smooth and wider-spaced riblet surfaces in Figs. 5.1 and 5.8, respectively. The closer spanwise proximity of the hairpin vortices would promote a cancelling effect of their spanwise component of induced motion, resulting in the concentrated presence of the high-speed streaks with larger perturbation velocity magnitude at the base of the riblet grooves. This allows the high-speed streaks to extend well downstream of the spot as is observed through 


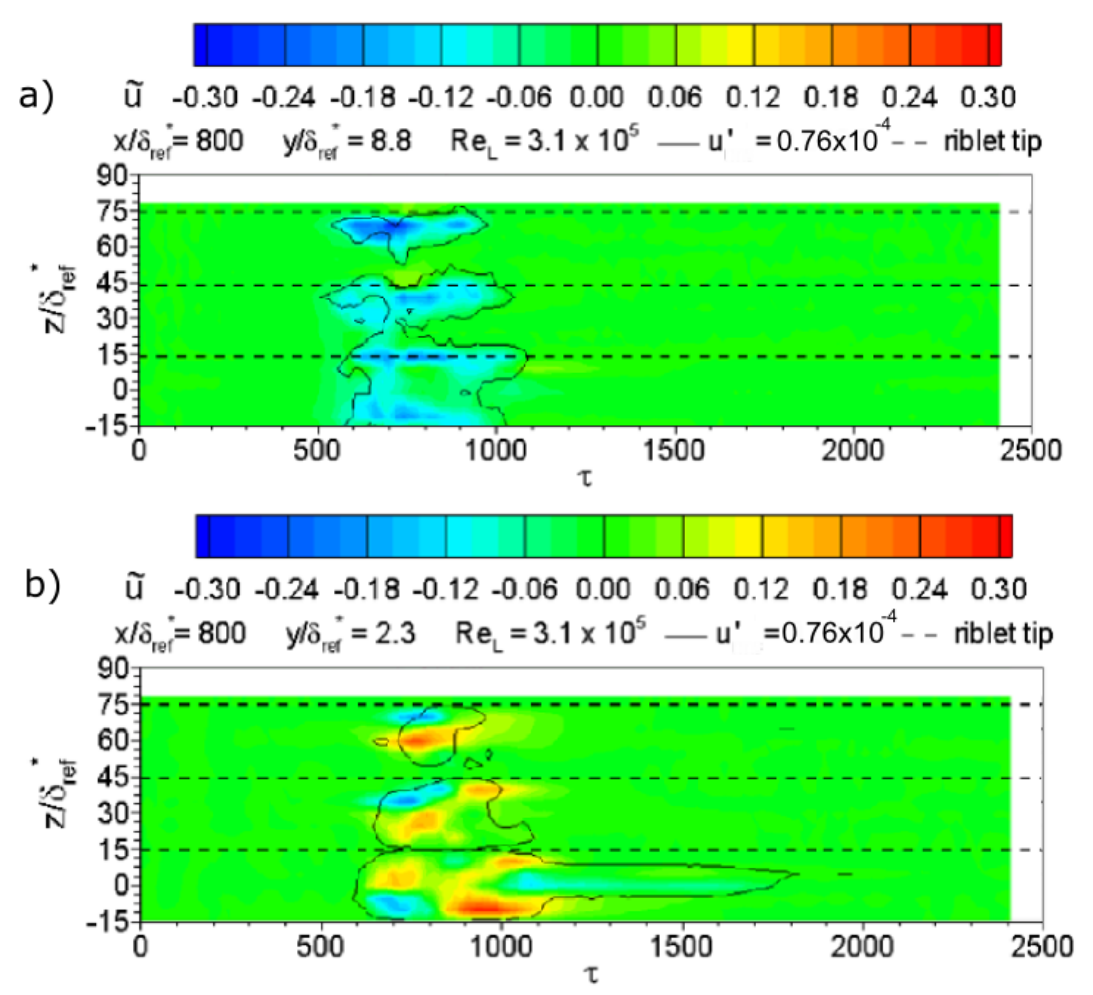

Figure 5.8: Topology of the turbulent spot developing over the wider-spaced riblet surface at a) $y / \delta_{r e f}^{*}=8.8$ and b) $y / \delta_{r e f}^{*}=2.3\left(R e_{L}=3.1 \times 10^{5}, \mathrm{x}=600 \mathrm{~mm}\right)$

the perturbation velocity in the y-t plane in Fig. 5.10 where the calmed region begins at $\tau=1500$ and extends past $\tau=2000$, nearly double the length observed over the smooth surface. The concentrated high-speed streaks, and the reduced streamwise momentum of the undisturbed fluid within the grooves both contribute to the larger streamwise dimension of the calmed region.

The wave packets over the closer-spaced riblet surface are positioned on the riblet tips as is evidenced by the positioning of the low-speed streaks in Fig. 5.6b, similar to the observations for the wider-spaced riblet surface. In this case, the low-speed streaks are observed to be even stronger than for the surface with wider-spaced riblets. This is suggestive of the presence of mechanisms that complement the effect of the riblets in inhibiting spanwise meandering of the streaks. One plausible mechanism is the lower streamwise momentum of the fluid in the narrower grooves of this surface that feeds the streaks via the upwash induced by the hairpin vortices straddling the riblet tips. The magnitude of the perturbation velocity 


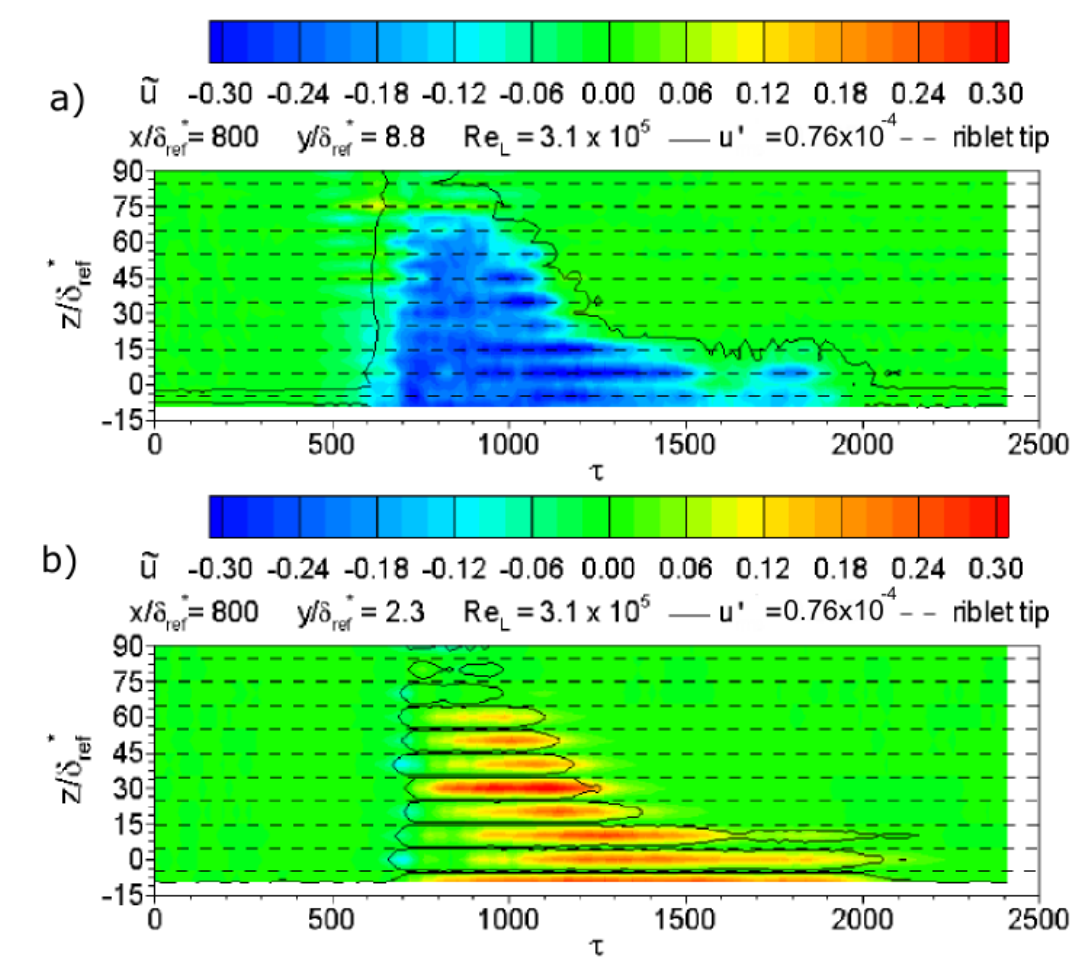

Figure 5.9: Topology of the turbulent spot over the closer-spaced riblet surface at a) $y / \delta_{r e f}^{*}=8.8$ and b) $y / \delta_{r e f}^{*}=2.3\left(R e_{L}=3.1 \times 10^{5}, \mathrm{x}=600 \mathrm{~mm}\right)$

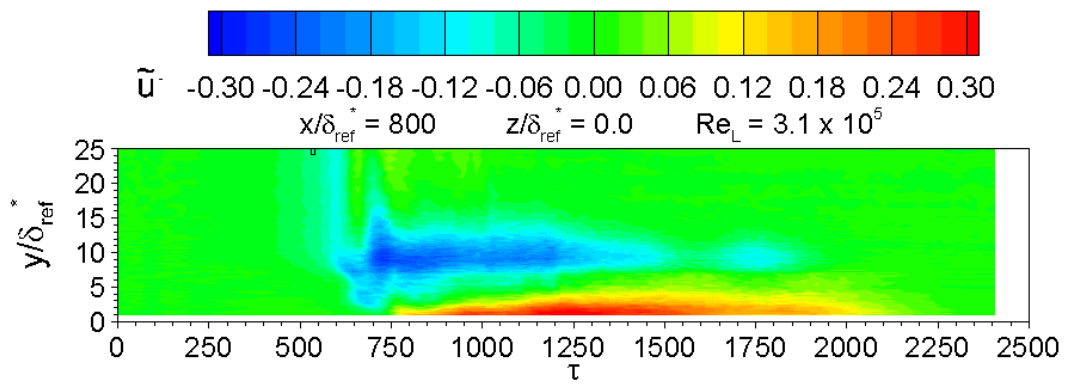

Figure 5.10: Perturbation velocity of the turbulent spot in the y-t plane on the closer-spaced riblet surface $\left(\operatorname{Re}_{L}=3.1 \times 10^{5}, \mathrm{x}=600 \mathrm{~mm}\right)$

of the streaks exceeds the threshold which supports the varicose mode of instability of the streaks (Elofsson et al., 1999; Brandt \& Henningson, 2002). However, examination of the instantaneous perturbation velocity field in the spanwise-wall normal plane reveals no periodic widening and narrowing of the low-speed streaks characteristic of the varicose instability mode. The lack of secondary instability is likely due to the stabilizing effect of the strong favourable streamwise pressure gradient imposed on the turbulent spot. 
The closer-spaced riblets are noted to yield twice as many streaks as for the smooth surface over the same spanwise extent. Unless the closer spanwise spacing of the wave packets and their hairpin vortices promotes destructive mutual interaction, one may expect the riblets to have an enhancing effect on the turbulent mixing. Some spanwise interaction of hairpin vortices that is promoted by the close spacing of the riblets is evident through regions of negative perturbation velocity spanning between adjacent low-speed streaks. These low-speed regions are likely the result of induced motions of spanwise vortices which form spanning the grooves similar to the observations of Garcia-Mayoral \& Jimenez (2011a) over riblets spaced 50 wall-units apart. Garcia-Mayoral and Jimenez noted that the strength of the spanwise vortices increases greatly with riblet size, thus the large riblets of the present study could feasibly create strong enough spanwise vortices which could span grooves of 100 wall-unit width and induce wall-normal motions leading to the observed low-speed regions. The formation of these vortices is promoted by the inflection point in the streamwise velocity profile within the riblet groove (Fig. 5.5b) which likely undergoes $\mathrm{K}-\mathrm{H}$ instability as was observed by Garcia-Mayoral \& Jimenez (2011a). It is likely that the spanwise vortices forming over the grooves would connect with the nearby hairpin vortices leading to the pairing of adjacent hairpin vortices as depicted in Fig. 5.11. The spanwise vortices were observed to occur only in the presence of the wave packets in the turbulent spot, and were absent in the undisturbed boundary layer. This suggests that the velocity field induced by the wave packets appears to modify the shear layer residing over the grooves in a manner which is necessary for the formation of the spanwise vortices. The noted effects of the riblets on the hairpin vortices and their mutual interaction must also have been present during the early stages of development of the spot under the influence of the wake of the jet triggering the spot, resulting in the notably larger size of the spot in this case.

The relative size and growth of the turbulent spots over all three surfaces and at both flow Reynolds numbers are compared in Fig. 5.12 through the spot propagation parameter. As was observed for the smooth-surface case, the early development of the turbulent spot over the riblet surfaces is strongly influenced by the wake of the jet as evidenced by the larger spot propagation parameter at the most upstream measurement position. The spot propagation 


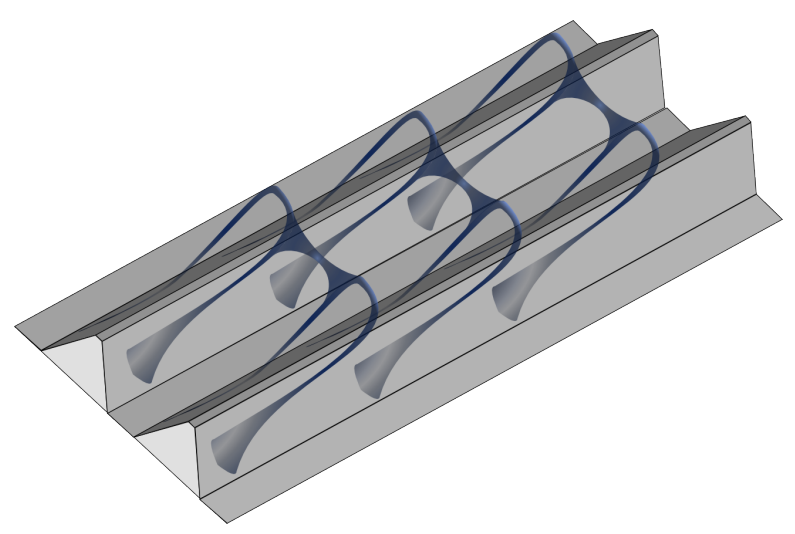

Figure 5.11: Schematic of two spanwise adjacent wave packets interacting with each other and the prevailing spanwise vortices over the surface with closer-spaced riblets

parameter for $R e_{L}=3.1 \times 10^{5}$ is larger for all three surfaces due to the weaker streamwise pressure gradient which does not have as strong of a stabilizing effect on the spot compared to the $R e_{L}=2.2 \times 10^{5}$ case. The turbulent spot over the surface with closer-spaced riblets has a significantly larger propagation parameter than either the smooth or wider-spaced-riblet surfaces which have similar propagation parameters. This trend is consistent with the stronger vortical dynamics within the spot for the surface with closer-spaced riblets.

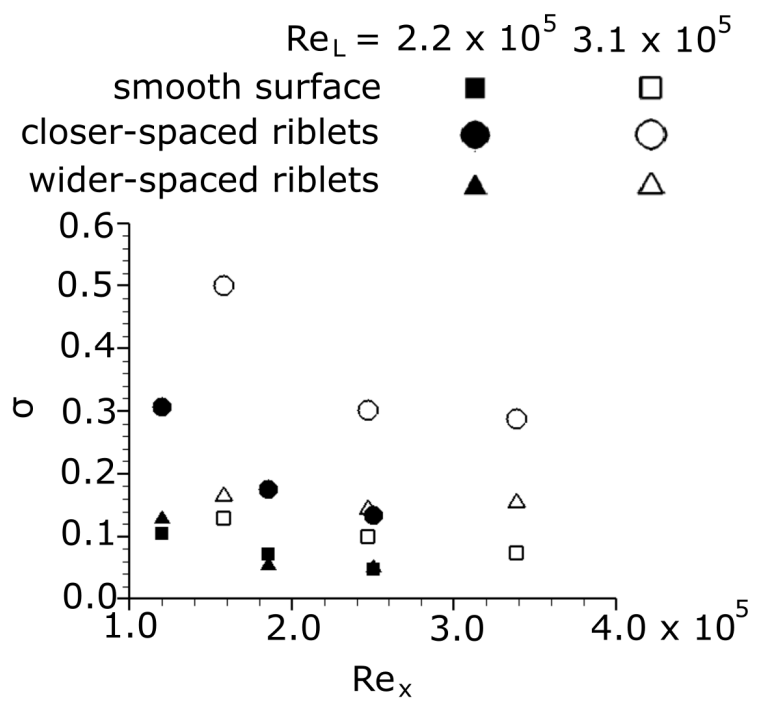

Figure 5.12: Streamwise variation of the spot propagation parameter over all three test surfaces at both flow Reynolds numbers 


\section{Chapter 6}

\section{Conclusions}

The velocity field in an artificially created turbulent spot over a smooth surface and two surfaces with streamwise riblets was examined experimentally. The experiments were performed on a flat surface with a strongly favourable streamwise pressure gradient. The chosen spanwise spacings of the riblets of 100 and 300 wall units correspond to the configuration that is identified as widely-spaced riblets in published literature. The chosen riblet height of 75 wall units is notably larger than those of previous studies, and was so chosen to promote more effective control over the wave packets and their hairpin vortices.

The surface with riblet spacing of 300 wall-units promoted spanwise spacing of wave packets that is locked to the spacing of the riblets, resulting in the wave packets to be more sparsely populated along the spanwise direction than on the smooth surface. This control on the spanwise positioning of the wave packets would also be expected to influence the sinuous mode of instability of the low-speed streaks, thus altering the turbulence regeneration mechanism.

The surface with riblet spacing of 100 wall-units had an even more pronounced effect in controlling the spanwise positioning of the wave packets. The results suggest notably stronger sweep and ejection events compared to those with the wider-spaced riblets and improved control of the spanwise meandering of the low speed streaks. Additionally, there is evidence of an additional instability mechanism resulting in the formation of spanwise-oriented vortical structures that interact with the hairpin vortices, thus contributing to the wall-normal mixing process. 
The control of coherent structures within the turbulent spot achieved by the riblet surfaces shows promise as an initial step towards the optimization of these surfaces for different purposes. The closer spanwise spacing of the hairpin vortices over the closer-spaced riblet surface, their increased mutual interaction, and the resultant increases in the strengths of the low- and high-velocity streaks collectively suggest that the riblets with closer spacing may have the net effect of promoting turbulence in an organized manner. Whether this can be optimized to realize enhancement in convection heat transfer with only modest increase in skin-friction drag would be worth examining further. In contrast, the wider-spaced-riblet surface shows potential in reducing the density of coherent structures and their corresponding velocity and pressure fluctuations. This may result in a reduction in flow-induced noise, and possibly lower skin friction drag and surface heat transfer if the reduction in turbulent mixing is not more-than-offset by the increased surface area. 


\section{References}

Adrian, R., Meinhart, C., \& Tomkins, C. (2000). Vortex organization in the outer region of the turbulent boundary layer. Journal of Fluid Mechanics, 422, pp. 1-54.

Amini, J. \& Lespinard, G. (1982). Experimental study on an "incipient spot" in a transitional boundary layer. Physics of Fluids, 25, pp. 1743-1750.

Asai, M., Minagawa, M., \& Nishioka, M. (2002). The instability and breakdown of a near-wall low-speed streak. Journal of Fluid Mechanics, 455, pp. 289-314.

Asai, M. \& Nishioka, M. (1989). Origin of the peak-valley wave structure leading to wall turbulence. Journal of Fluid Mechanics, 208, pp. 1-23.

Baig, M. \& Chernyshenko, S. (2004). Regeneration mechanism of streaks in near-wall quasi-2d turbulence. European Journal of Mechanics B/Fluids, 23, pp. 727-736.

Bechert, D., Bruse, M., Hage, W., der Hoeven, J. V., \& Hoppe, G. (1997). Experiments on drag-reducing surfaces and their optimization with an adjustable geometry. Journal of Fluid Mechanics, 338, pp. 59-87.

Bechert, D., Hage, W., \& Brusek, M. (1996). Drag reduction with the slip wall. AIAA Journal, 34, pp. 1072-1074.

Boiko, A. (2007). Effect of riblets on the streaky structures excited by free stream tip vortices in boundary layer. Journal of Mechanical Science and Technology, 21, pp. 196-206.

Brandt, L. \& Henningson, D. (2002). Transition of streamwise streaks in zero-pressuregradient boundary layers. Journal of Fluid Mechanics, 472, pp. 229-261. 
Brinkerhoff, J. \& Yaras, M. (2012). Direct numerical simulation of a square jet ejected transversely into an accelerating, laminar main flow. Flow Turbulence and Combustion, 89, 519-546.

Brinkerhoff, J. \& Yaras, M. (2014). Numerical investigation of the generation and growth of coherent flow structures in a triggered turbulent spot. Journal of Fluid Mechanics, 759, pp. 257-294.

Brinkerhoff, J. \& Yaras, M. (2015). Numerical investigation of transition in a boundary layer subjected to favourable and adverse streamwise pressure gradients and elevated free stream turbulence. Journal of Fluid Mechanics, 781, pp. 52-86.

Bruun, H. (1995). Hot-wire anemometry principles and signal analysis. Oxford University Press.

Bushnell, D. (2003). Aircraft drag reduction - a review. Proceedings of the Institution of Mechanical Engineers, Part G: Journal of Aerospace Engineering.

Bushnell, D. \& McGinley, C. (1989). Turbulence control in wall flows. Annual Reviews in Fluid Mechanics, 21, pp. 1-20.

Butler, K. \& Farrell, B. (1993). Optimal perturbations and streak spacing in wall-bounded turbulent shear flow. Physics of Fluids, 5, pp. 774-777.

Chernorai, V., Kozlov, V., Loefdahl, L., Grek, G., \& Chun, H. (2006). Effect of riblets on nonlinear disturbances in the boundary layer. Thermophysics and Aeromechanics, 13(1).

Choi, K.S., D. J. \& Clayton, B. (1998). Turbulent boundary-layer control by means of spanwise-wall oscillation. AIAA Journal, 36, pp. 1157-1163.

Choi, H., Moin, P., \& Kim, J. (1993). Direct numerical simulation of turbulent flow over riblets. Journal of Fluid Mechanics, 255, pp. 503-539. 
Choi, K., Yang, X., Clayton, B., Glover, E., Atlar, M., Semenov, B., \& Kulik, V. (1997). Turbulent drag reduction using compliant surfaces. Proceedings: Mathematical, Physical and Engineering Sciences, 453, pp. 2229-2240.

Clark, C. (2009). Development of a low-speed wind tunnel and performance evaluation of the exhaust duct of a hydrokinetic turbine. M.A.Sc. thesis, Carleton University.

Dixon, A., Lucey, A., \& Carpenter, P. (1994). Optimization of viscoelastic compliant walls for transition delay. AIAA Journal, 32, pp. 256-267.

Durst, F. \& Zanoun, E. (2002). Experimental investigation of near-wall effects on hot-wire measurements. Experiments in Fluids, 33, pp. 210-218.

Elofsson, A., Kawakami, M., \& Alfredsson, P. (1999). Experiments on the stability of streamwise streaks in plane poiseuille flow. Physics of Fluids, 11(1), pp. 915-930.

Fric, T. \& Roshko, A. (1994). Vortical structure in the wake of a transverse jet. Journal of Fluid Mechanics, 279, pp. 1-47.

Gadelhak, M., Blackwelder, R., \& Riley, J. (1981). On the growth of turbulent regions in laminar boundary layers. Journal of Fluid Mechanics, 110, pp. 73-95.

Garcia-Mayoral, R. \& Jimenez, J. (2011a). Drag reduction by riblets. Philosophical transactions: Mathematical, Physical and Engineering Sciences, 369(1940), pp. 1412-1427.

Garcia-Mayoral, R. \& Jimenez, J. (2011b). Hydrodynamic stability and breakdown of the viscous regime over riblets. Journal of Fluid Mechanics, 678, pp. 317-347.

Goldstein, D., Handler, R., \& Sirovich, L. (1995). Direct numerical simulation of turbulent flow over a modelled riblet covered surface. Journal of Fluid Mechanics, 302, pp. 333-376.

Goldstein, D. \& Tuan, T. (1998). Secondary flow induced by riblets. Journal of Fluid Mechanics, 363, pp. 115-151.

Gostelow, J., Melwani, N., \& Walker, G. (1996). Effects of streamwise pressure gradient on turbulent spot development. ASME J. Turbomach., 118, pp. 737-743. 
Groth, J. \& Johansson, A. (1988). Turbulence reduction by screens. Journal of Fluid Mechanics, 197, pp. 139-155.

Gutmark, E. \& Blackwelder, R. (1987). On the structure of a turbulent spot in a heated laminar boundary layer. Experiments in Fluids, 5, pp. 217-229.

Hack, M. \& Zaki, T. (2014). Streak instabilities in boundary layers beneath freestream turbulence. Journal of Fluid Mechanics, 741, pp. 280-315.

Jacobs, R. \& Durbin, P. (2001). Simulations of bypass transition. Journal of Fluid Mechanics, 428, pp. $185-221$.

Johnson, M. (2001). On the flow structure within a turbulent spot. International Journal of Heat and Fluid Flow, 22, pp. 409-416.

Jones, V. \& Launder, B. (1972). Some properties of sink-flow turbulent boundary layers. Journal of Fluid Mechanics, 56, pp. 337-351.

Kachanov, Y. (1994). Physical mechanisms of laminar-boundary-layer transition. Annual Reviews in Fluid Mechanics, 26, pp. 411-482.

Katz, Y., Seifert, A., \& Wygnanski, I. (1990). On the evolution of the turbulent spot in a laminar boundary layer with a favourable pressure gradient. Journal of Fluid Mechanics, 221 , pp. 1-22.

Keith, W. (1989). Spectral measurements of pressure fluctuations on riblets. AIAA Journal, 27(12), pp. 1822-1824.

Kline, S., Reynolds, W., Schraub, F., \& Runstadler, P. (1983). The structure of turbulent boundary layers. Journal of Fluid Mechanics, 30, pp. 741-773.

Klumpp, S., Meinke, M., \& Schroder, W. (2010). Numerical simulation of riblet controlled spatial transition in a zero-pressure-gradient boundary layer. Flow, Turbulence and Combustion, 85, pp. 57-71. 
Lancey, T. \& Reidy, L. (1989). Effects of surface riblets on the reduction of wall pressure fluctuations in turbulent boundary layers. J. Acoust. Soc. Am., 85(4), pp. 1793-1794.

Lee, S. \& Lee, S. (2001). Flow field analysis of a turbulent boundary layer over a riblet surface. Experiments in Fluids, 30, pp. 153-166.

Litvinenko, Y., Chernoray, V., Kozlov, V., Loefdahl, L., Grek, G., \& Chun, H. (2006). The influence of riblets on the development of a lambda structure and its transformation into a turbulent spot. Doklady Physics, 51, pp. 144-147.

Ma, H., Tian, Q., \& Wu, H. (2005). Experimental study of turbulent boundary layers on groove/smooth flat surfaces. Journal of Thermal Science, 14(3), pp. 193-197.

Mohammadi, A. \& Floryan, J. (2013a). Groove optimization for drag reduction. Physics of Fluids, 25(113601).

Mohammadi, A. \& Floryan, J. (2013b). Pressure losses in grooved channels. Journal of Fluid Mechanics, 725, pp. 23-54.

Narasimha, R. (1985). The laminar-turbulent transition zone in the boundary layer. Prog. Aerospace Sciences, 22, pp. 29-80.

Panton, R. (2001). Overview of the self-sustaining mechanisms of wall turbulence. Progress in Aerospace Sciences, 37, pp. 341-383.

Perry, A., Lim, T., \& Teh, E. (1981). A visual study of turbulent spots. Journal of Fluid Mechanics, 104, pp. 387-405.

Piomelli, U., Balaras, E., \& Pascarelli, A. (2000). Turbulent structures in accelerating boundary layers. Journal of Turbulence, 1.

Piomelli, U. \& Yuan, J. (2013). Numerical simulations of spatially developing, accelerating boundary layers. Physics of Fluids, 25(101304).

Reed, H. \& Saric, W. (1996). Linear stability theory applied to boundary layers. Annual Reviews in Fluid Mechanics, 28, pp. 389-428. 
Roberts, S. \& Yaras, M. (2004). Boundary-layer transition over rough surfaces with elevated free-stream turbulence. Proceedings of ASME Turbo Expo 2004, GT2004-53668.

Roberts, S. \& Yaras, M. (2005). Modeling transition in separated and attached boundary layers. ASME Journal of Turbomachinery, 127, pp. 402-411.

Sabatino, D. \& Smith, C. (2008). Turbulent spot flow topology and mechanisms for surface heat transfer. Journal of Fluid Mechanics, 612, pp. 81-105.

Samimy, M., Kim, J., Kastner, J., Adamovich, I., \& Utkin, Y. (2007). Active control of highspeed and high-reynolds-number jets using plasma actuators. Journal of Fluid Mechanics, 578, pp. 305-330.

Scheiman, J. \& Brooks, J. (1980). Comparison of experimental and theoretical turbulence reduction from screens, honeycomb, and honeycomb-screen combinations. Journal of Aircraft, 18(8), pp. 638-643.

Schröder, A. \& Kompenhans, J. (2004). Investigation of a turbulent spot using multi-plane stereo particle image velocimetry. Experiments in Fluids, 36, pp. 82-90.

Schubauer, G. \& Klebanoff, P. (1956). Contributions on the mechanics of boundary-layer transition. Technical Report 1289, NACA.

Schubauer, G., Spangernberg, W., \& Klebanoff, P. (1950). Aerodynamic characteristics of damping screens. Technical report, NACA.

Singer, B. \& Joslin, R. (1994). Metamorphosis of a hairpin vortex into a young turbulent spot. Physics of Fluids, 6, pp. 3724-3736.

Smith, C. \& Metzler, S. (1983). The characteristics of low-speed streaks in the near-wall region of a turbulent boundary layer. Journal of Fluid Mechanics, 129, pp. 27-54.

Smits, A. \& Delo, C. (2001). Self sustaining mechanisms of wall turbulence. Lecture notes in Physics, (pp. pp. 17-38). 
Stalio, E. \& Nobile, E. (2003). Direct numerical simulation of heat transfer over riblets. International Journal of Heat and Fluid Flow, 24, pp. 356-371.

Strand, J. \& Goldstein, D. (2011). Direct numerical simulations of riblets to constrain the growth of turbulent spots. Journal of Fluid Mechanics, 668, pp. 267-292.

Suga, K., Mori, M., \& Kaneda, M. (2011). Vortex structure of turbulence over permeable walls. International Journal of Heat and Fluid Flow, 32, pp. 586-595.

Tomkins, C. \& Adrian, R. (2003). Spanwise structure and scale growth in turbulent boundary layers. Journal of Fluid Mechanics, 490, pp. 37-54.

Wu, X., Jacobs, R., Hunt, J., \& Durbin, P. (1999). Simulation of boundary layer transition induced by periodically passing wakes. Journal of Fluid Mechanics, 398, pp. 109-153.

Wygnanski, I., Sokolov, M., \& Friedman, D. (1976). On a turbulent 'spot' in a laminar boundary layer. Journal of Fluid Mechanics, 78, pp. 785-819.

Yaras, M. (2007). An experimental study of artificially-generated turbulent spots under strong favorable pressure gradients and freestream turbulence. Journal of Fluids Engineering, 129, pp. $563-572$.

Zhong, S., Kittichaikan, C., Hodson, H., \& Ireland, P. (1998). Visualisation of turbulent spots under the influence of adverse pressure gradients. Experiments in Fluids, 28, pp. 385-393.

Zhou, J., Adrian, R., Balachandar, S., \& Kendall, T. (1999). Mechanisms for generating coherent packets of hairpin vortices in channel flow. Journal of Fluid Mechanics, 387, pp. 353-396. 


\section{Appendix A}

\section{Instrumentation Details}

\section{A.1 Data acquisition system}

The traverse system and instrumentation on the wind tunnel in 3175 Mackenzie Building at Carleton University are connected to a single National Instruments NIDAQ-USB 6356 data acquisition device (DAQ). The DAQ has an internal buffer of 32 million samples and is capable of simultaneously sampling eight analog input channels through dedicated 16-bit analog-to-digital (A/D) converters at a rate of up to $1.25 \mathrm{MHz}$ per channel. Table A.1 shows the analog input voltage range settings available and their associated measurement resolution. The resolution of the signal is insensitive to the sampling rate or the number of channels being sampled either in sequence or simultaneously.

Table A.1: A/D Conversion Resolution

\begin{tabular}{cc}
\hline Input Range & A/D Conversion Resolution \\
\hline$-10 \mathrm{~V}$ to $10 \mathrm{~V}$ & $320 \mu \mathrm{V}$ \\
$-5 \mathrm{~V}$ to $5 \mathrm{~V}$ & $160 \mu \mathrm{V}$ \\
$-2 \mathrm{~V}$ to $2 \mathrm{~V}$ & $64 \mu \mathrm{V}$ \\
$-1 \mathrm{~V}$ to $1 \mathrm{~V}$ & $32 \mu \mathrm{V}$ \\
\hline
\end{tabular}

A test of the analog input accuracy was conducted in order to identify and eliminate any sources of environmental signal noise. These tests were conducted by applying a constant DC voltage in the form of a AA battery connected to a 10 -turn $10 \mathrm{k} \Omega$ potentiometer which was 
sampled at a high rate to resolve any high frequency noise in the signal. Analog input channels were sampled individually as well as in groups of up to four channels sampled simultaneously.

Several sources of signal noise were identified and removed during this process. Signal noise was found to be created by the traverse system stepper motor drivers and their power supplies and the variable-frequency drive (VFD) used to control the wind tunnel fan. To reduce the signal noise from these sources, the stepper motor drivers and power supplies were moved to a different shelf in the instrumentation cabinet as far from the DAQ as possible. The VFD was placed in the corner of the laboratory approximately $3 \mathrm{~m}$ away from the instrumentation cabinet, reducing the noise levels in the sampled analog sample.

Signal noise was also found to occur if the cabinet was plugged into the same power bar as the computer interfacing with the DAQ. To eliminate this noise source, the DAQ was setup to draw power from the main cabinet power bar which was in turn connected directly to the building power on the same building supply circuit. Signal noise was created when the analog signal was simultaneously being measured by the DAQ and a multimeter. Finally, it was found that sampling analog signals that were connected to adjacent analog channels on the DAQ caused a small amount of signal noise. All measurements were taken with at least one disconnected analog channel in between analog signals when possible.

With all sources of signal noise removed as per the foregoing, the uncertainty in measurements caused by the DAQ is of the order of the quantization error of the 16-bit A/D converter presented in Table A.1.

\section{A.2 Pressure transducers}

A total of ten pressure transducers are located in the instrumentation cabinet. These consist of two MKS Instruments 220DD Baratron differential capacitance transducers (Model \# 220DD-00010A2B) and eight Honeywell DC capacitive transducers (Model \# DC002NDR5). The calibration coefficient was determined for each pressure transducer by correlating the output voltage of the pressure transducers with measurements taken with an inclined manometer. The inclined manometer was set at an inclination of 1:5 and filled with red manometer fluid 
(S.G. 0.826). The inclined manometer was marked at $1 \mathrm{~mm}$ intervals, resulting in a measurement precision of $\pm 0.17 \mathrm{mmH}_{2} \mathrm{O}$. The calibration coefficient was taken as the slope of a least squares line fit with an $\mathrm{R}$ value of 0.9588 of the pressure measured by the inclined manometer versus the output voltage of each transducer and is presented in Table A.2. The pressure simultaneously measured by the inclined manometer and the pressure transducer was increased using a clamp which was moved incrementally up a length of tubing attached simultaneously to one of the measurement ports of the pressure transducer and the manometer while the other port of the two instruments remained open to the laboratory atmosphere. Ten data points were measured with the clamp attached to tubing on each pressure port of the pressure transducer resulting in a total of 20 points for the calibration. While the Honeywell transducers were calibrated, they were not used in experiments. The Baratron pressure transducers were used for all measurements during experimentation.

Table A.2: Pressure transducer calibration coefficients

\begin{tabular}{cc}
\hline \multicolumn{2}{c}{ MKS Baratron Transducer } \\
\hline I.D. & Calibration Coefficient $(\mathrm{Pa} / \mathrm{mV})$ \\
\hline 293468 & $0.130 \pm 0.001$ \\
874204 & 0.130 \\
\hline \multicolumn{2}{c}{ Honeywell DC Transducer } \\
\hline I.D. & Calibration Coefficient $(\mathrm{Pa} / \mathrm{mV})$ \\
\hline T0 & $0.193 \pm 0.001$ \\
T1 & 0.193 \\
T2 & 0.194 \\
T3 & 0.193 \\
T4 & 0.194 \\
T5 & 0.193 \\
T6 & 0.193 \\
T7 & 0.193 \\
\hline
\end{tabular}




\section{A.3 Thermocouple}

A J-type thermocouple was used to measure the local air temperature. The leads of the thermocouple were attached to the stem of the hotwire probe just downstream of the removable portion of the probe during both calibration of the hotwire and during experiments. The thermocouple was calibrated against a mercury thermometer with a precision of one degree Celsius. The calibration was done by submerging both the thermocouple and the thermometer in a cup of water, the temperature of which was increased incrementally by adding hot water from another cup. The voltage output of the thermocouple was converted into a temperature using a third order polynomial in the data acquisition software and was compared to the temperature measured by the thermometer. The converted thermocouple temperature reading was found to have a linear relationship with the temperature measured by the thermometer and therefore a correction equation was incorporated into the data acquisition software (Fig. A.1). The uncertainty of the temperature measurement was found to be $\pm 0.5^{\circ} \mathrm{C}$.

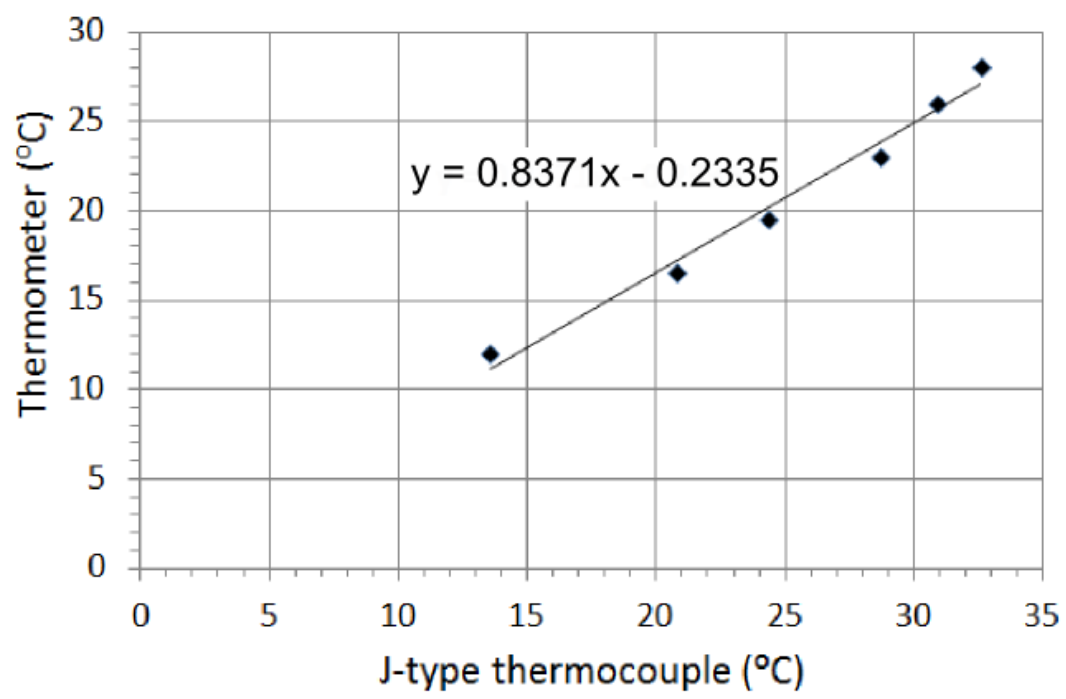

Figure A.1: Thermocouple calibration 


\section{A.4 Hotwire anemometer}

Experiments were conducted with a single-sensor hotwire probe connected to an AN-1003 constant temperature anemometer. The probe had a $1.3 \mathrm{~mm}$ long sensor made of $5 \mu \mathrm{m}$ diameter tungsten wire. An overheat ratio of 1.76 was used resulting in a sensor temperature of $225^{\circ} \mathrm{C}$. The frequency response of the $\mathrm{AN}-1003$ was optimized such that velocity fluctuations up to $12 \mathrm{kHz}$ could be measured. The data acquisition software used a ninth-order polynomial to convert the anemometer output voltages to flow velocity. The polynomial was fitted to 20 points evenly distributed between speeds of 0 and $12 \mathrm{~m} / \mathrm{s}$ using a TSI Air Velocity Calibrator. The calibration was repeated 16 times and the average polynomial was used (Fig. A.2). The measurement uncertainty was found to be $\pm 2 \%$ except for speeds below $0.5 \mathrm{~m} / \mathrm{s}$ which had an uncertainty of $\pm 5 \%$, both at a $99 \%$ confidence interval. The uncertainty in the velocity measurement was primarily due to electronic drift of the AN-1003 signal-conditioning circuitry which caused a slight drift in the polynomial fitted to each of the 16 data sets.

The drift in the ambient temperature in the laboratory was compensated for using the correlation suggested by Bruun (1995):

$$
E_{w}=E_{w, r}\left(\frac{T_{w}-T_{a}}{T_{w}-T_{a, r}}\right)^{1 / 2}
$$

where $E_{w}$ is the temperature corrected hotwire signal, $E_{w, r}$ is the raw hotwire signal, $T_{w}$ is the wire temperature, $T_{a}$ is the ambient temperature, and $T_{r, a}$ is the reference ambient temperature. This correlation is accurate for temperature changes up to $5^{\circ} \mathrm{C}$ in the temperature range of $10^{\circ} \mathrm{C}$ to $80^{\circ} \mathrm{C}$.

Measuring flow velocity near a wall with a hotwire probe creates several potential sources of error. The mitigation of this error was done in a manner consistent with the literature on the subject and is discussed in Chapter 3. A source of error that is exacerbated by the topology of the measurement surfaces being investigated is the blockage effect of the probe stem when measuring the flow within the valley of the grooves. The large size of the probe relative to the opening of the valley required a sensitivity study on whether the 10 degree inclination used in the baseline case was sufficient to mitigate the blockage effect of the probe 


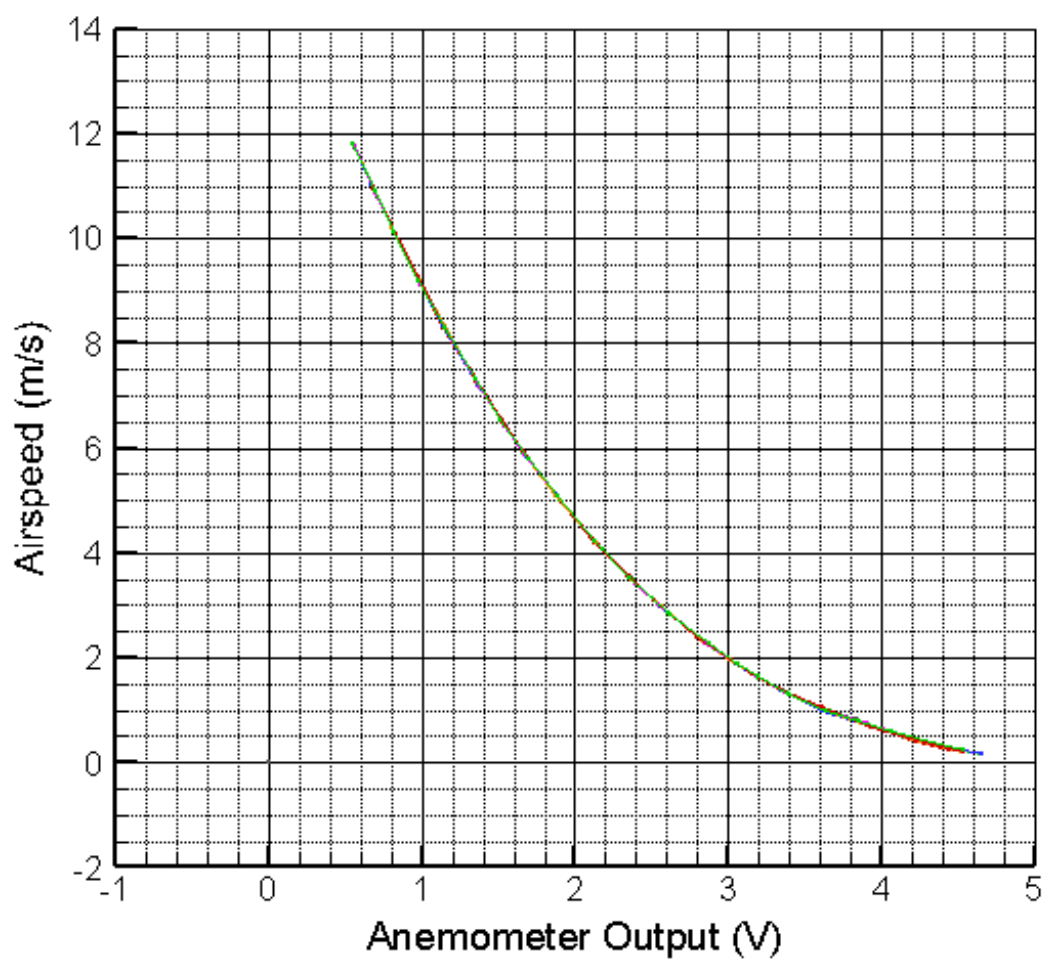

Figure A.2: Results of 16 calibrations of the hotwire sensor approximated by a 9th-order polynomial fit, each colour represents one calibration curve

stem during measurements on the modified surfaces. The sensitivity study was conducted by measuring the undisturbed boundary layer in the valley of the groove at $\mathrm{x}=600 \mathrm{~mm}$ and $\mathrm{z}$ $=0 \mathrm{~mm}$ of the closer-spaced riblet surface at three probe orientations: two with the sensor parallel to the base of the groove with the probe stem inclined at 10 degrees and 30 degrees, respectively, and one with the sensor normal to the base of the groove at with the probe stem inclined at 10 degrees. Before each measurement, the probe was calibrated at the corresponding sensor orientation and probe stem inclination. Another traverse was conducted with an S-shaped, boundary-layer pitot probe with a thickness much smaller than the groove width and which had sufficient reach to fully remove the probe body from the groove. Static pressure was measured from the $P_{c, 2}$ port of the wind tunnel which, while not equal to the static pressure at the measurement location, provided a steady reference point from which to determine the relative dynamic pressure between measurement points within the boundary 
layer. The resulting dynamic pressure was normalized by the freestream value measured by the pitot probe at the streamwise location of the traverse. Fig. A.3 shows the resulting boundary-layer velocity profiles for each case with the square of the local velocity relative to the freestream velocity at the streamwise location of the traverse on the $\mathrm{x}$-axis so that the hotwire velocity measurements could be directly compared to the pitot probe dynamic pressure measurements. Comparing the undisturbed boundary-layer velocity profiles it was determined that a 10 degree orientation with the probe parallel to the wall was suitable to avoid any blockage effects in the groove.

a)

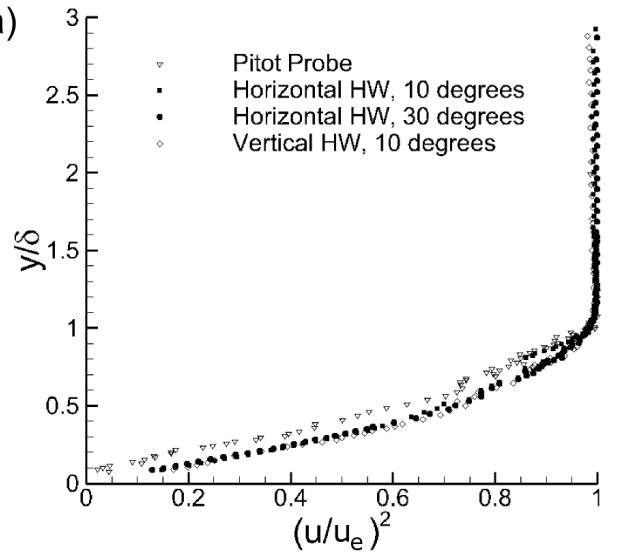

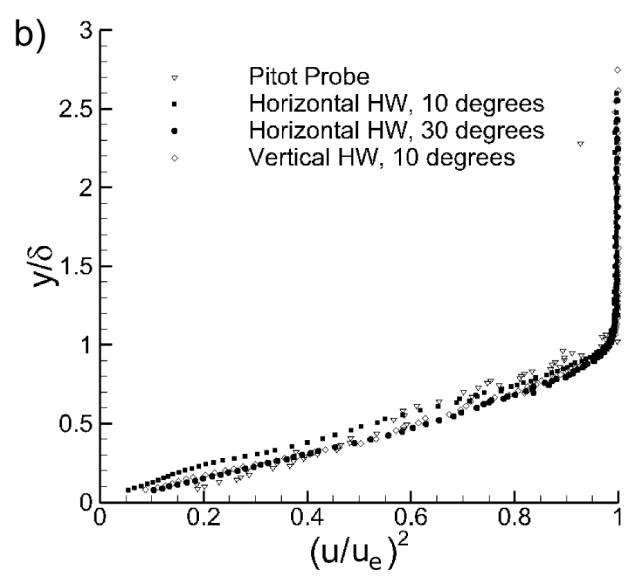

Figure A.3: Probe angle sensitivity study for a) $R e_{L}=2.2 \times 10^{5}$ and b) $R e_{L}=3.1 \times 10^{5}$ at $\mathrm{x}=600 \mathrm{~mm}, \mathrm{z}=0 \mathrm{~mm}$ on the closer-spaced riblet surface 


\section{Appendix B}

\section{Wind Tunnel Commissioning}

The configuration of the wind-tunnel used in the present study is shown in Fig. B.1. Following construction in 2009, several measurements were taken to quantify the quality of the flow entering the test section (Clark, 2009). Several changes have been made to the wind tunnel since the original commissioning was done including the addition of acoustic panels immediately downstream of the outlet of the fan section, upgrading of the flow conditioning screen at the contraction inlet, and the implementation of new data acquisition and traverse systems. These changes required the wind tunnel be recommissioned prior to beginning any experiments. The results of the commissioning tests for the data acquisition system, traverse system and contraction outlet flow are outlined in this appendix along with the results of benchmark tests.

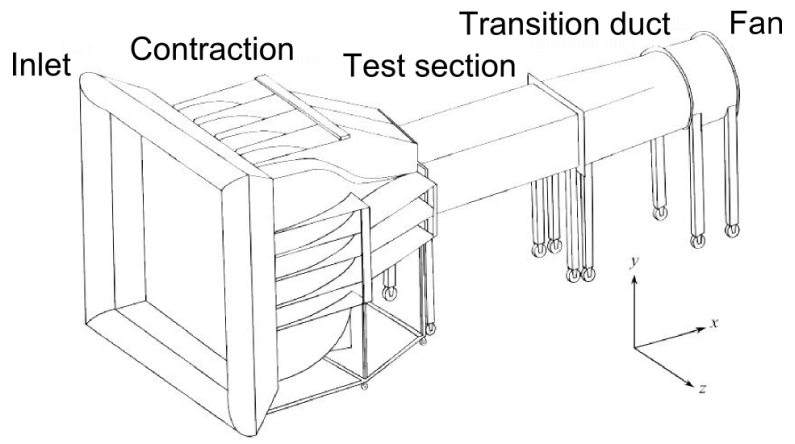

Figure B.1: Schematic of Carleton University's low-turbulence, open-circuit, suction wind tunnel 
Table B.1: Traverse system commissioning data

\begin{tabular}{cccccc}
\hline Axis & $\begin{array}{c}\text { Pulse Freq } \\
(\mathrm{kHz})\end{array}$ & $\begin{array}{c}\text { Dist/Step } \\
(\mu \mathrm{m})\end{array}$ & $\begin{array}{c}\text { Min. Traverse } \\
(\mathrm{mm})\end{array}$ & $\begin{array}{c}\text { Accuracy } \\
( \pm \mathrm{mm})\end{array}$ & $\begin{array}{c}\text { Backlash } \\
(\mathrm{mm})\end{array}$ \\
\hline Streamwise & 4 & 198 & 0.050 & 0.013 & 0.151 \\
\hline Spanwise & 80 & 197 & 0.025 & 0.015 & 0.000 \\
\hline Vertical & 90 & 197 & 0.025 & 0.017 & 0.000 \\
\hline
\end{tabular}

\section{B.1 Traverse system commissioning}

A three-axis traverse system is suspended from the ceiling of the laboratory over the test section of the wind tunnel and is used to move the probe stem during experiments. All three axes are traversed using lead screws turned by stepper motors. There is one stepper motor for each of the vertical and spanwise axes, and two stepper motors for the streamwise axis. The stepper motor drivers receive digital pulses through the digital output ports in the DAQ and send signals to the stepper motor dictating the frequency and number of steps commanded by the data acquisition software. Prior to commissioning the traverse system, tests were performed to determine the highest possible pulse frequency which the motors were capable of while maintaining movement precision (Table B.1).

The accuracy of the traverse motion in all three axes was determined by repeatedly traversing the probe stem and comparing the distance commanded through the data acquisition software to the distance traversed as measured using a dial gauge with a resolution of $0.0254 \mathrm{~mm}$. The dial gauge was placed near where the probe stem was held by the traverse system to eliminate errors caused by bending of the stem. Traverses were conducted over distances between $0.025 \mathrm{~mm}$ and $20.0 \mathrm{~mm}$, corresponding to the measurable range of motion of the dial gauge, and were repeated ten times for each axis. During each traverse the backlash was measured as the probe stem was brought back to its original position. As per Table B.1, the spanwise and vertical axes were able to perform movements as small as $0.025 \mathrm{~mm}$ with no backlash, while the streamwise axis could only perform traverses greater than $0.050 \mathrm{~mm}$ and had a backlash of $0.151 \mathrm{~mm}$. 


\section{B.2 Contraction outlet flow commissioning with an empty test section}

The flow uniformity, turbulence levels and the condition of the boundary layer of the flow exiting the contraction were measured in order to determine the baseline flow entering the test section during experiments. As shown in the schematic of the laboratory in Fig. B.2, the tight placement of the wind tunnel in the laboratory requires that the placement of components be done precisely. The distances between the contraction inlet and the walls of the lab have been shown to have a significant effect on the flow uniformity entering the test section (Clark, 2009). In accordance with the recommendations provided by Clark (2009), the distances $\Delta 1$ and $\Delta 2$ identified in Fig. B.2 are set to $0.34 \mathrm{~m}$ and $0.36 \mathrm{~m}$ respectively.

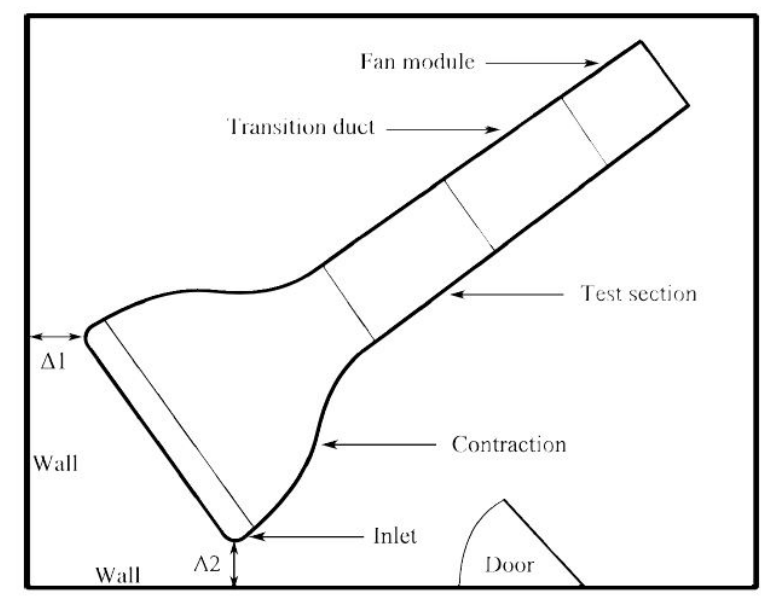

Figure B.2: Positioning of the wind tunnel within the Laboratory

To further improve the flow uniformity and to reduce the intensity of the freestream turbulence flow conditioning screens consisting of a layer of furnace filter and two layers of nylon mesh of differing mesh specifications was installed. The furnace filter was draped over the contraction inlet upstream of the bell-mouth and served to filter large-scale transient flows present in the lab. The nylon meshes were installed between the bell-mouth and the contraction such that the two nylon meshes were in direct contact with each other. The screens were tensioned by hanging a steel L-bracket from their base and securing the top of the screens to the top of the contraction. The straps attaching the bell-mouth to the inlet 
were tightened to grip the nylon mesh so that it did not move when the wind tunnel was in operation. A shadowgraph of each nylon mesh is shown in Fig. B.3. The meshes that were used were the Standard Mosquito Mesh and Shade Screen provided by Mosquito Curtains. The Standard Mosquito Mesh has an open area ratio of $64 \%$ and a wire diameter of $0.33 \mathrm{~mm}$. The Shade Screen is made up of alternating open and closed cells in a checkerboard pattern and has an open area ratio of $20 \%$. While the open area ratios of meshes are quite small, the resulting turbulence reduction is not equal to the equivalent metal meshes (Groth \& Johansson, 1988; Scheiman \& Brooks, 1980; Schubauer et al., 1950). The flexibility of the nylon allows the openings to widen and the wires to contract when under tension, thus increasing the open area and reducing the turbulence damping ability of the screen.

a)

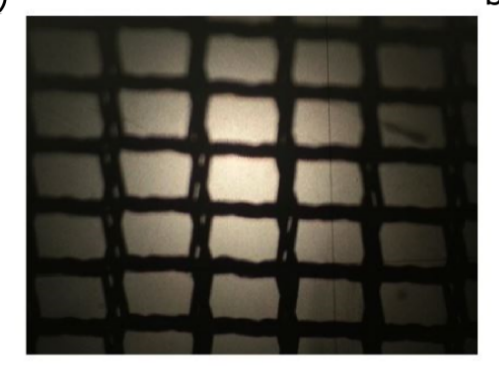

b)

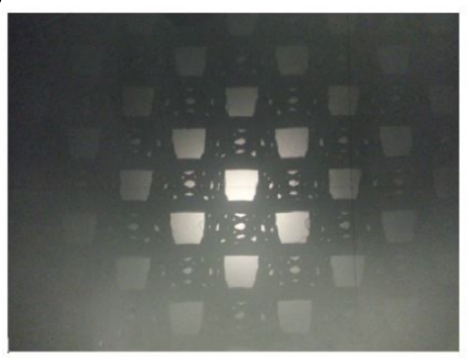

Figure B.3: Shadowgraph of the a) Standard Mosquito Mesh and b) Shade Screen

The commissioning tests described in this section were performed with an empty test section. All measurements were taken at centreline velocities of $4.5 \mathrm{~m} / \mathrm{s}$ and $9.0 \mathrm{~m} / \mathrm{s}$ and in the vertical-spanwise plane $50.8 \mathrm{~mm}$ upstream of the contraction outlet, which corresponded to the streamwise location of the $P_{c, 2}$ static pressure tap, unless otherwise stated.

\section{B.2.1 Contraction wall boundary layer at the outlet}

The boundary-layer velocity profile at the wall of the contraction was measured to ascertain the state of the boundary layer as the flow entered the test section. The measurements were performed using the hotwire probe on the lower wall of the contraction outlet $190 \mathrm{~mm}$ from the centreline and $50.8 \mathrm{~mm}$ upstream of the contraction outlet plane. The boundary layer was traversed in the wall-normal direction over 80 measurement positions concentrated near the 
wall and starting $0.1 \mathrm{~mm}$ above the surface to resolve the prevailing high velocity gradients. The hotwire was sampled at $8192 \mathrm{~Hz}$ for one second at each position and the samples were averaged over time. The boundary layer velocity profiles had shape factors of 2.5 and 2.1 for the low and high speed cases respectively, indicating an attached, laminar boundary layer exiting the contraction.

\section{B.2.2 Contraction outlet flow uniformity}

The uniformity of the flow exiting the contraction was measured using a pitot-static probe. The probe was traversed over a grid with $25.4 \mathrm{~mm}$ spacing between measurement locations extending to within $25.4 \mathrm{~mm}$ of wall proximity. The pressure transducer output was sampled at $150 \mathrm{~Hz}$ over 4500 samples. As shown in Fig. B.4 show that the velocity varies less than $2 \%$ over the majority of the contraction outlet at both operating conditions.

\section{B.2.3 Flow alignment}

The direction of the incoming flow was determined using the four-hole pressure probe described in Chapter 3 placed at the centreline of the contraction outlet. Geometrically opposing pressure taps of the probe were sampled differentially at $150 \mathrm{~Hz}$ sampling frequency over 750 samples as the probe was rotated in one degree increments in the pitch and yaw axes separately between \pm 5 degrees from its reference position parallel to the walls of the test section. After completing a traverse in one of the axes, the probe was rotated 180 degrees about its longitudinal axis and the process was repeated. A line of best fit was plotted for each traverse as shown in Figs. B.5 and B.6, and the flow angle was determined from the point of intersection of the two lines. The flow at the exit of the contraction was determined to have pitch angles of $2.1 \pm 0.1$ and $1.8 \pm 0.1$ degrees at $4.5 \mathrm{~m} / \mathrm{s}$ and $9.0 \mathrm{~m} / \mathrm{s}$, respectively, where a positive angle indicates the flow is moving towards the lower portion of the flow path. The yaw angle in both cases was $1.5 \pm 0.1$ degrees from the right when facing upstream. 

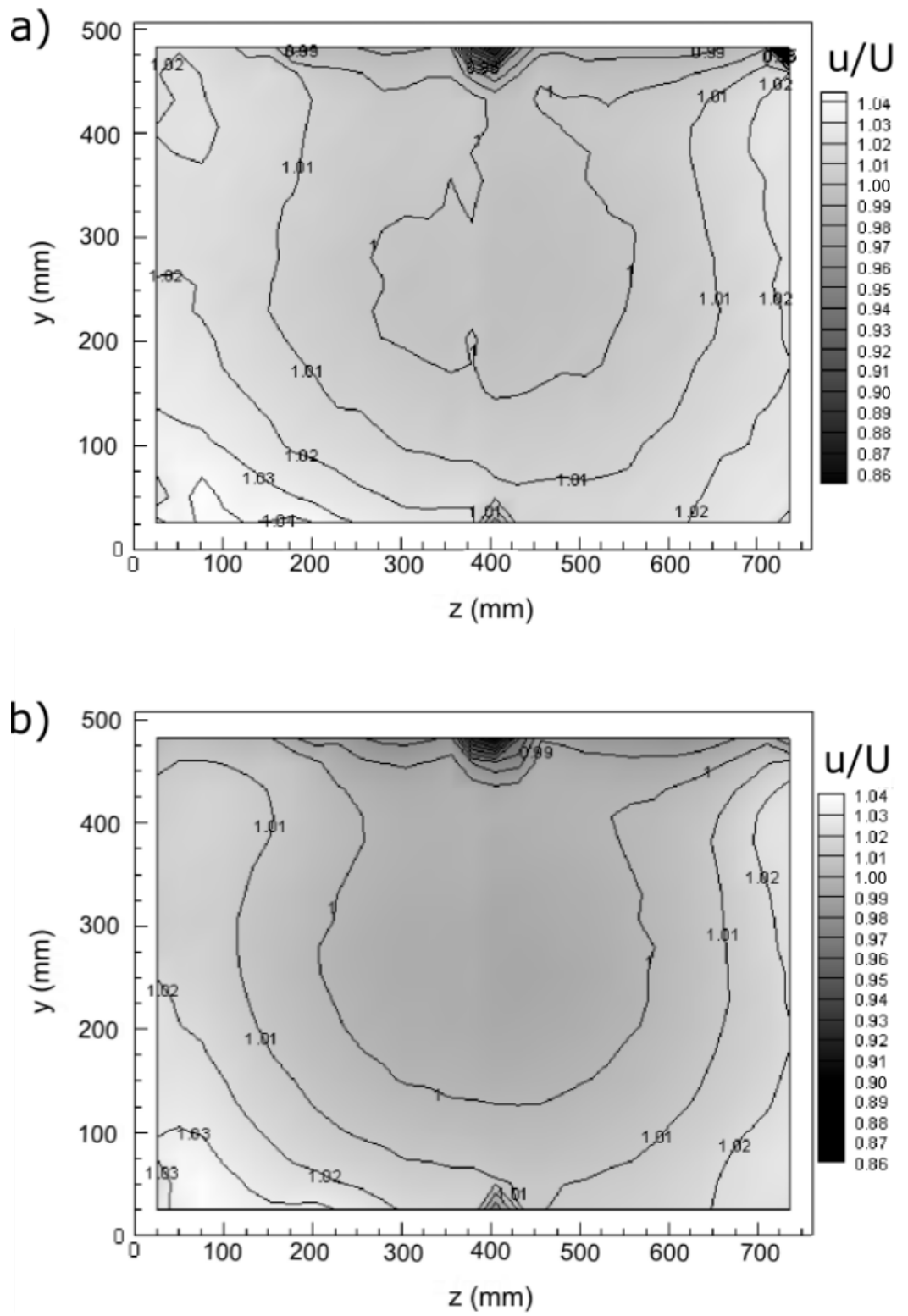

Figure B.4: Flow uniformity at the contraction outlet for a) $4.5 \mathrm{~m} / \mathrm{s}$ and b) $9.0 \mathrm{~m} / \mathrm{s}$ centreline velocity

\section{B.2.4 Freestream turbulence}

The freestream turbulence of the flow exiting the contraction was measured using a singlesensor hotwire probe placed at the centreline of the contraction outlet. The probe was aligned in the direction of the flow exiting the contraction and the centreline velocity was varied from 

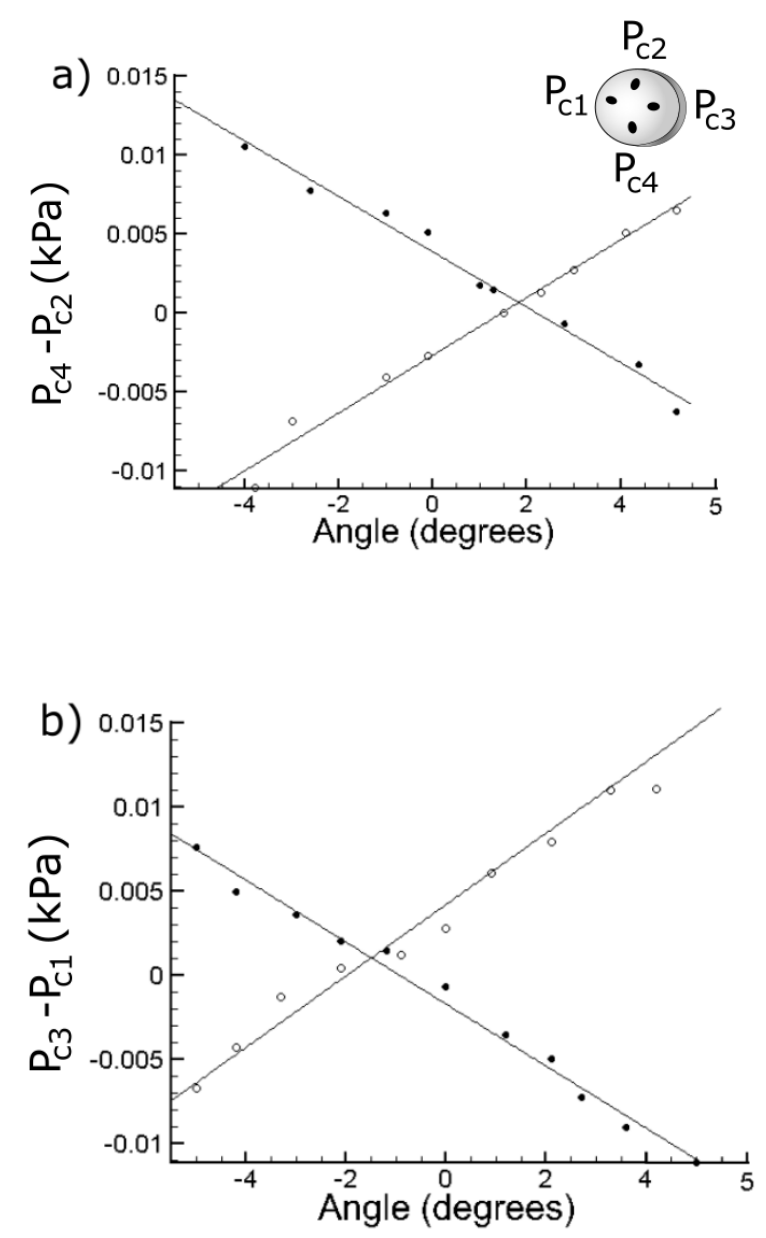

Figure B.5: Contraction outlet flow alignment in a) pitch and b) yaw for $9.0 \mathrm{~m} / \mathrm{s}$

$1 \mathrm{~m} / \mathrm{s}$ to $10 \mathrm{~m} / \mathrm{s}$. The turbulence was measured once with the hotwire sensor oriented to be sensitive to streamwise and vertical velocity fluctuations, and again with the sensor oriented to be sensitive to streamwise and spanwise velocity fluctuations at $12 \mathrm{kHz}$ over 60,000 samples. The turbulence intensity, $T u \%=100 \times \frac{u^{\prime}}{U}$, for each centreline velocity, shown in Fig. B.7, is noted to be less than $0.75 \%$ at all operating conditions and at both sensor orientations, where $u^{\prime}$ is the RMS velocity fluctuation and $U$ is the time-averaged freestream velocity. The integral length scale of the turbulence was calculated by performing an autocorrelation of the velocity fluctuations and assuming Taylor's frozen eddy hypothesis. The length scale of the turbulence was found to be on the order of the height of the test section at all velocities. 

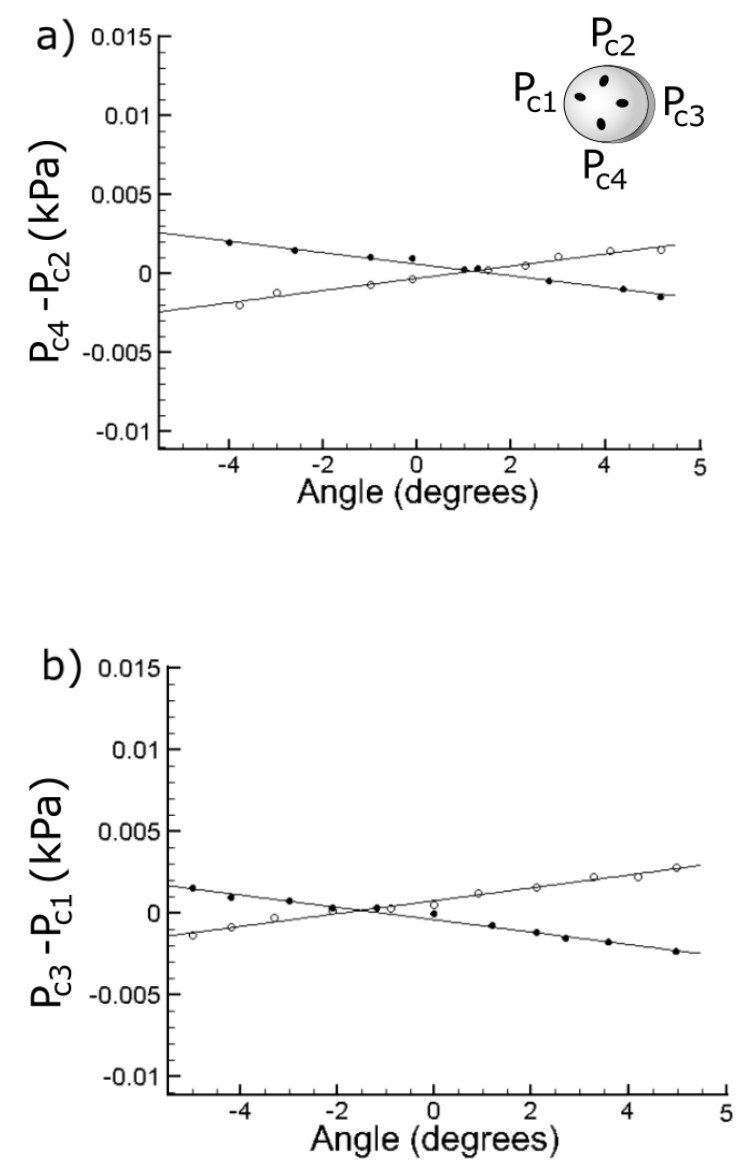

Figure B.6: Contraction outlet flow alignment in a) pitch and b) yaw for $4.5 \mathrm{~m} / \mathrm{s}$

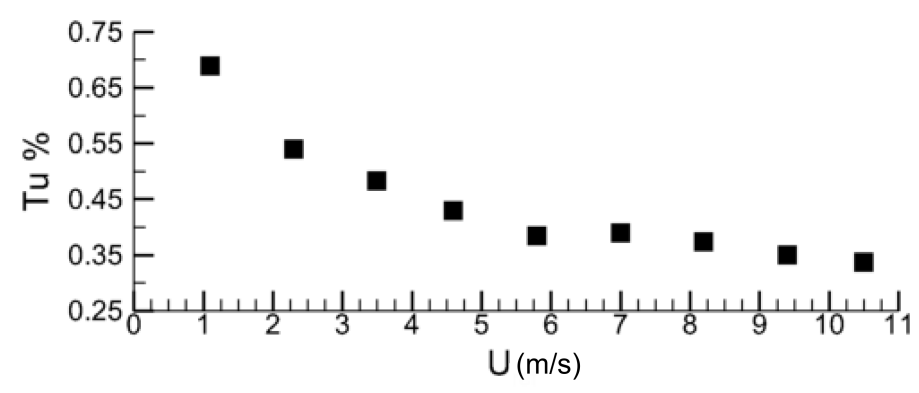

Figure B.7: Contraction outlet freestream turbulence intensity at various wind tunnel centreline velocities 


\section{B.3 Benchmark testing}

In order to verify the accuracy of the instrumentation system and to validate the proper functioning of the wind-tunnel, a benchmark test was undertaken to reproduce the Blasius boundary layer before continuing with experimentation. In order to reproduce the Blasius flow, a flat plate made of MDF with an aluminum leading edge as described in Chapter 3 was installed in the test section. A flap was attached to the ceiling above the trailing edge of the plate and was adjusted such that the stagnation point was shifted slightly towards the working side of the test plate. The boundary layer at freestream velocities of $2.2 \mathrm{~m} / \mathrm{s}, 4.6 \mathrm{~m} / \mathrm{s}$ and $9.5 \mathrm{~m} / \mathrm{s}$ were traversed using a hotwire probe at 8 streamwise locations approximately $100 \mathrm{~mm}$ apart between $65 \mathrm{~mm}$ and $800 \mathrm{~mm}$ downstream of the leading-edge of the test surface and at 45 wall-normal positions clustered towards the surface. The anemometer signal was sampled at $12 \mathrm{kHz}$ for five seconds at each position. 
a)

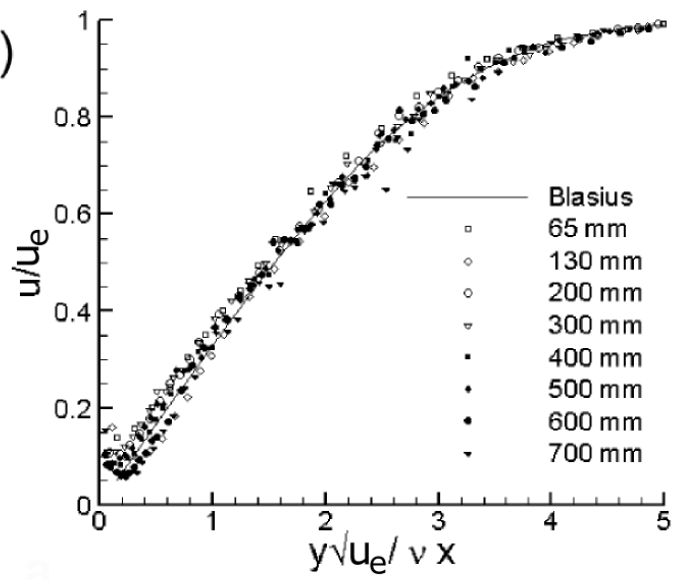

b)

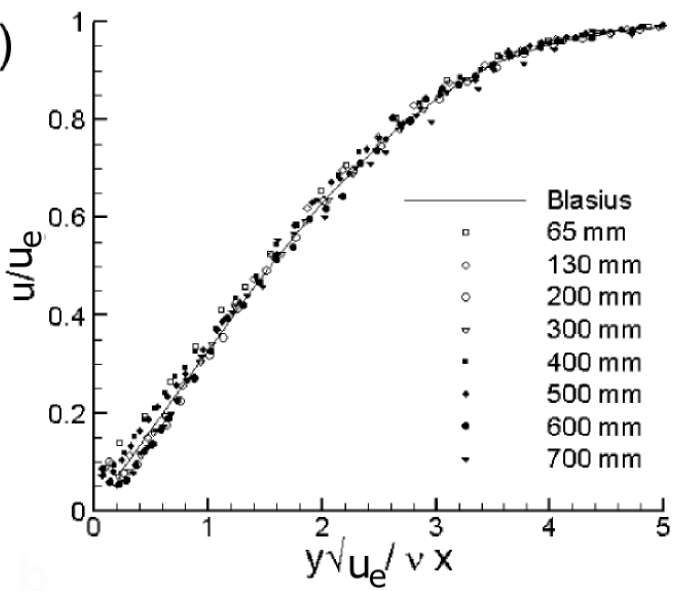

c)

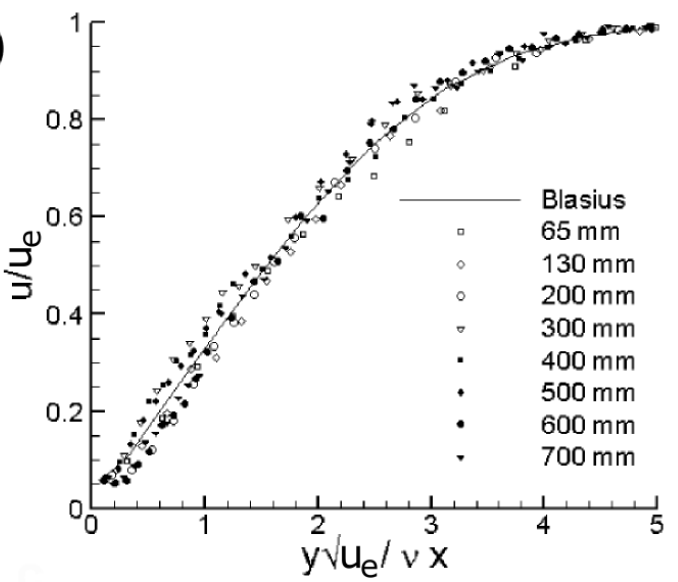

Figure B.8: Benchmark measurement of the Blasius boundary layer at a) $2.2 \mathrm{~m} / \mathrm{s}$, b) $4.6 \mathrm{~m} / \mathrm{s}$ and c) $9.5 \mathrm{~m} / \mathrm{s}$ 


\section{Appendix C}

\section{Additional Information on the Experimental Setup}

The experimental setup was concisely described in Chapter 3 with the goal of providing the reader with sufficient background to understand the work that was undertaken, and give context to the results presented. This appendix contains further details of the experimental setup so that it can be more easily repeated by future researchers.

This appendix describes the methods used to manufacture the test surfaces and provides a more detailed description of the experimental setup, and the setup and duration of the jet for the smooth surface case.

\section{C.1 Surface manufacturing}

The two riblet surfaces used in the experiments were manufactured using a custom cutting tool designed to match the cross-section of the closer-spaced riblet surface. A CNC router was used to remove material from a sheet of one half inch thick MDF. The most significant challenge in manufacturing the surfaces was avoiding breaking the tips of the riblets. It was found that using a sharp bit was essential to avoiding tip breakage and that each bit could only create one or two surfaces before becoming too dull due to the abrasive nature of MDF. A bit speed of 18000 RPM and traverse speeds no higher than 150 inches/min were found to consistently produce good quality grooves. It was found that the best way to cut the grooves was to do so in a single pass at full groove depth instead of using several shallower cuts. The single pass method ensured that there was supporting material around the groove peak as 
it was cut which prevented it from becoming chipped. The surface finish was found to be adequately smooth using this method. The lack of supporting material at the end of the groove cuts resulted in the downstream edge of the groove tips being broken apart. Since the measurements were done far upstream of the breakage point, it was decided that this defect was acceptable but should be resolved for future work.

Each surface had several threaded inserts installed on its underside to provide attachment points for the aluminum leading edge, the solenoid valve, and the upper flap. The jet orifice was drilled using a \# 69 drill bit and a small portable drill press. The jet orifice was drilled in a single pass in order to ensure repeatability of the orifice geometry.

\section{C.2 Test section setup}

The one-quarter-inch thick polycarbonate ceiling was inclined at 13 degrees to the test surface and was supported using six threaded rods through holes in the ceiling of the test section. The rods were adjusted using nuts and washers to ensure a constant angle along the length of the ceiling. A 0.040 -inch-thick sheet of polycarbonate was attached to the leading edge of the ceiling using silicone and then to the test section ceiling at the inlet using clear packing tape. This ensured a smooth transition from the horizontal ceiling of the test section to the angled polycarbonate ceiling.

To prevent separation at the downstream edge of the ceiling, a diffuser was installed in the fan duct to more gradually expand the flow into the fan. The diffuser consisted of a

sheet of plywood the upstream edge of which was aligned with the downstream edge of the ceiling, and was angled upwards at 5.6 degrees to reach just over half of the fan diameter at its downstream edge. The downstream edge of the plywood was supported by a 12 inch tall aluminum pillar with a 4 inch by 4 inch square cross-section beneath the plywood.

The freestream streamwise acceleration and the maximum freestream velocity in the test section were found to be sensitive to the positioning of the test surface relative to the ceiling. In each test case of the study, the leading edge of the test surface was $336 \mathrm{~mm}$ from the test section inlet and the upper surface was $175 \mathrm{~mm}$ above the floor. The surface was elevated 
using a welded steel frame and aluminum spacers. Washers were used at the corners of the base to level the surface. Foam insulation tape was installed on the spanwise edges of the test surface to prevent flow leaking over the sides of the surface. This positioning resulted in an area ratio of 10.3 between the leading and trailing edges of the test surface.

\section{C.3 Jet}

The jet disturbance was triggered by a solenoid valve which released air from the building high-pressure air supply through the jet orifice to create the disturbance which would result in the turbulent spot. The solenoid valve was triggered using a pulse generator connected to an amplifier. The pulse had a square wave-form with a duration of $9.6 \mathrm{~ms}$ and a frequency of 1.25 Hz. The amplifier was set to the minimum setting required to reliably open the solenoid valve to minimize the duration of the jet. The resulting jet had a duration of $50 \mathrm{~ms}$ with a velocity of approximately $60 \mathrm{~m} / \mathrm{s}$ which were measured by placing the hotwire probe $25 \mathrm{~mm}$ above the jet orifice and measuring several trigger cycles. The duration of the jet pulse was determined using the results shown in Fig. C.1 from the point the anemometer output registers the jet to the point where the recovery back to undisturbed flow becomes non-linear. This end-point was chosen because the non-linear region of the velocity trace is caused by fluid entrained by the jet and does not reflect the actual jet duration at the wall. The jet velocity was determined by calibrating the probe to $16 \mathrm{~m} / \mathrm{s}$ which is the highest velocity possible using the probe calibrator. The last 5 points of the calibration curve were used to linearly extrapolate the velocity at lower anemometer voltages, hence the stated jet velocity is an approximate value. 


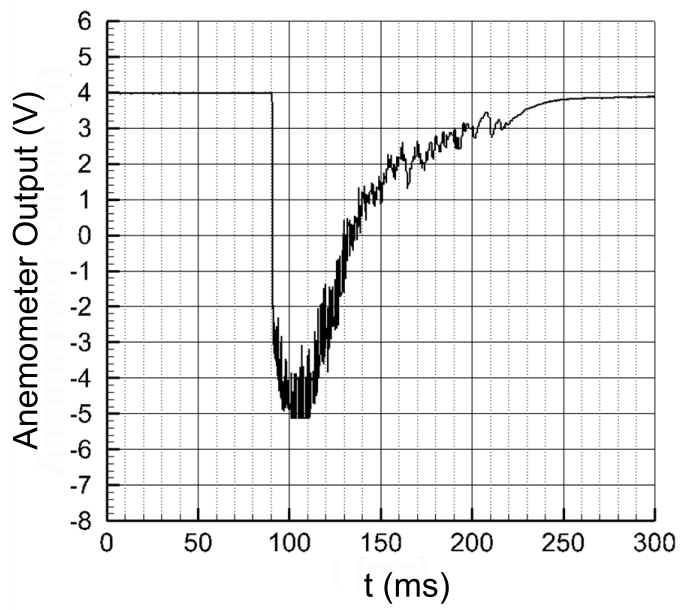

Figure C.1: Anemometer signal of jet measurement $25 \mathrm{~mm}$ above the smooth surface 